\title{
Betrachtungen zur Frequenzplanung für terrestrisches Fernsehen in den 50er Jahren in Mitteleuropa
}

\section{U. Kühn}

Ring 61, 15754 Haidsee O.T. Kolberg, Germany

Zusammenfassung. Der Bericht beleuchtet die Entwicklung der Fernseh- und Rundfunknetze im Osten Deutschlands ab Anfang der 50er Jahre. Neben einer ausführlichen Darstellung der technischen Aspekte liegt ein weiterer Schwerpunkt auf der Darstellung der nötigen Zusammenarbeit mit den Arbeitsgruppen der angrenzenden Länder. Diese war, bedingt durch die politische Lage, gerade mit den Experten aus Westdeutschland oft schwierig. Die maßgeblich beteiligten Personen und ihre Arbeiten werden vorgestellt. Der Autor war in leitender Position an der Entwicklung in Ostdeutschland beteiligt und liefert mit diesem Bericht einen aus technischer und historischer Sicht wertvollen Beitrag zur Entwicklung des Rundfunks.

\section{Einleitung}

Anfang der 50er Jahre begann man sich in Deutschland Gedanken über einen optimalen Aufbau der Fernsehnetze und über die Plannungsprinzipien für UKW-Hörrundfunk zu machen. Dabei wurde sehr schnell deutlich, dass beim Betreiben interferenzfreier Netze troposphärischen Überreichweiten eine gravierende Bedeutung zukommt, und diese daher entsprechend berücksichtigt werden müssen. Eigene Erkenntnisse, die über die von CCIR veröffentlichten Daten hinaus gingen, gab es zu dieser Zeit nur wenige. Es war daher dringend geboten, solche Unterlagen möglichst schnell und umfassend verfügbar zu machen.

Um diesen Erfordernissen nachzukommen, konnte im Westen Deutschlands auf das FTZ/Darmstadt und auf die technischen Abteilungen der Rundfunkanstalten zurückgegriffen werden. Dies war im Osten nicht gegeben. So kam es zur Gründung einer Außenstelle des damaligen Instituts für Postund Fernmeldewesen mit dem Sitz in Kolberg, etwa $60 \mathrm{~km}$

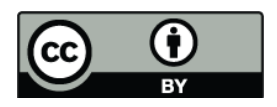

Correspondence to: M. Koch (michael.koch@fh-hannover.de) südöstlich von Berlin auf einer Geländeerhebung $92 \mathrm{~m}$ über NN. In der einsamen und elektromagnetisch störungsfreien Umgebung konnten Messungen von Wellenausbreitung und Funkentstörung gut durchgeführt werden.

Gegründet und geleitet wurde die Außenstelle von dem Physiker H. Kusch, der auch die Erfordernisse der Netzplanung schnell erkannte. Erste Vorarbeiten zur Frequenzplanung erfolgten durch den Mathematiker Apitz. Als der Verfasser 1955 seine Tätigkeit in Kolberg aufnahm, lagen erste Richtlinien für eine Frequenzplanung für UKW- und FSNetze bereits vor. Dafür als Grundlagen dienten die Arbeiten von Gressman und Kaltbeitzer, v. Rautenfeld und Fastert, Knöpfel und Stepp, sowie Nestel und Schwarz und Großkopf (Gressman und Kaltbeitzer, 1952; v. Rautenfeld und Fastert, 1952; Knöpfel und Stepp, 1953; Nestel und Schwarz, 1953, und Großkopf, 1952).

In der Praxis wurde meist das vereinfachte Multiplikationsverfahren verwendet, wobei anfangs die Rechnungen per Hand erfolgten, später wurden vom FTZ automatisierte Berechnungen angewandt, wodurch die gemeinsamen Arbeiten in Darmstadt erheblich beschleunigt wurden. Diese Arbeiten, die mit einem Treffen beim FTZ begannen, wurden im Osten maßgeblich angeregt und unterstützt durch den damaligen stellvertretenden Minister für Post- und Fernmeldewesen, Bereich Rundfunk und Fernsehen, Probst, der auch in der Folgezeit die Arbeiten unterstützte.

\section{Technische Arbeiten}

Das erste Treffen zweier Arbeitsgruppen zur Frequenzplanung aus Ost und West fand im FTZ Darmstadt statt. Die Organisation auf westlicher Seite lag bei H. Fleischer, die Delegation aus dem Osten wurde, soweit dies aus der Erinnerung des Verfassers hervorgeht, von E. Augustin, später von J. Brückner geleitet. Teilgenommen haben Mitarbeiter verschiedener westlicher Rundfunkanstalten, vor allem die 


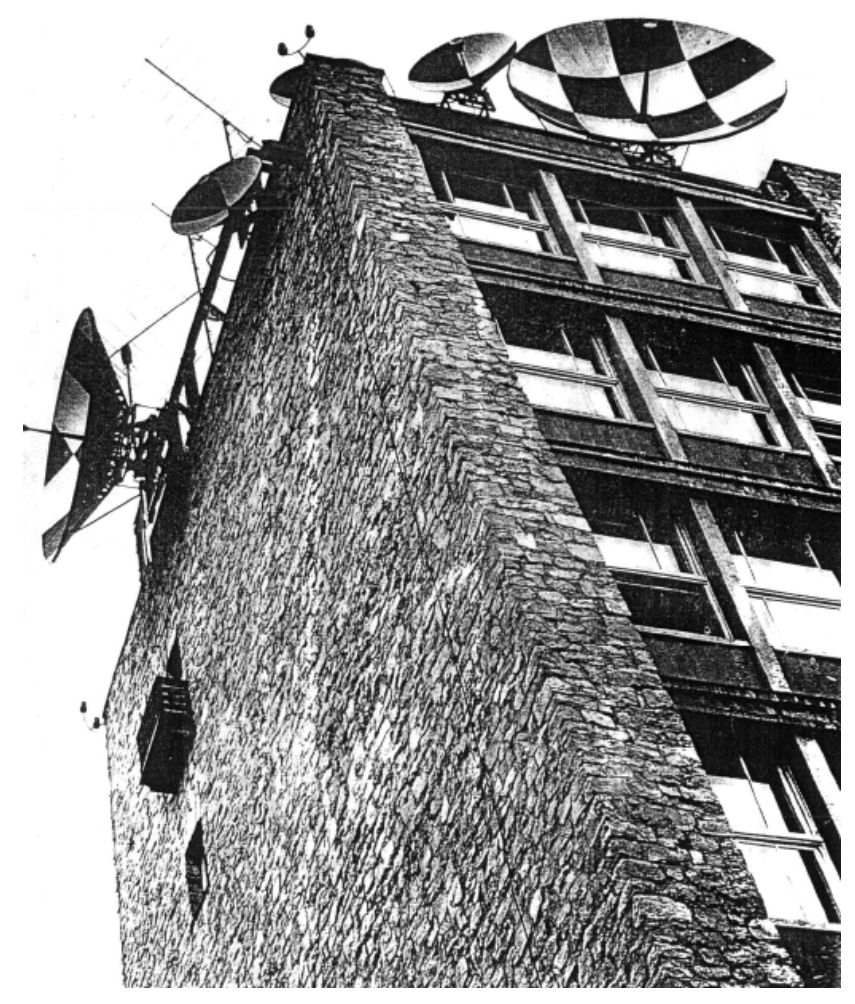

Abb. 1. Steinturm auf dem Kolberg an dem alle Antennen zur Messung troposphärischer Überreichweiten montiert waren.

Herren Stepp und Gutzmann traten aktiv in Erscheinung, ebenso wie der technische Direktor des SWF Knöpfel sowie andere Kollegen. Die Wellenausbreitung wurde aus westlicher Position von J. Großkopf und dessen Mitarbeiter Halbedel vertreten. In analoger Funktion betätigten sich U. Kühn und dessen Mitarbeiter H. Apitz.

Erschwerend für die Verhandlungen waren die unterschiedlichen Normen, besonders hinsichtlich des BildTonabstandes in Frankreich sowie den östlichen Ländern. Es erwies sich als unbedingt erforderlich, alle technischen Möglichkeiten einzubeziehen, die zu einer Verbesserung des Schutzabstandes beitrugen, wie off-set, Einsatz von Richtstrahlung, Leistungsbegrenzungen und Polarisationsentkopplung.

Es wurden sowohl Rechnungen zur Frequenzplanung durchgeführt, als auch Bestimmungen der Versorgungsgebiete. Wegen der zeitweise auftretenden Überreichweiten bei bestimmten meteorologischen Bedingungen mußten auch weit entfernte Sender berücksichtigt werden, wobei Material über die troposhärische Fernausbreitung weitgehend fehlte. Ionosphärische Überreichweiten, die im Band I stören könnten, wurden nicht berücksichtigt, darauf hatte man sich schnell geeinigt. Die reinen Rechenarbeiten erfolgten vorwiegend durch Mitarbeiter des FTZ. Die Atmosphäre war ausgezeichnet, und die Arbeiten schritten schnell voran. Von einer politisch gefärbten Einflußnahme war im Kreis der dort arbeitenden Fachkollegen zu keiner Zeit etwas zu bemerken, wobei es natürlich derartige Interessen im Hintergrund gab.

\section{Wellenausbreitung}

Bald hatte sich herausgestellt, dass die verwendeten Ausbreitungsdaten erhebliche Unsicherheiten enthielten. Vor allem die Ausbreitungskurven im Fernfeld aber auch im Nahbereich, die Geländedämpfung und die örtliche Feldstärkestreuung. So wurde anfangs mit einem einheitlichen, fixen Wert gearbeitet, der auf Messungen aus den USA zurückging. Erst viel später wurde festgestellt, welchen Veränderungen dieser Wert unterliegen kann. Im Westen erfolgten dann Arbeiten

- zur troposphärischen Fernausbreitung

- Feinstrukturuntersuchungen des Fernfeldes bei Troposcatter

Auf ostdeutscher Seite wurde verstärkt gearbeitet an

- Überreichweitenmessung bis $436 \mathrm{~km}$ (Feldberg/T. Kolberg)

- Örtliche Feldstärkestreuung und deren regionale Verteilung

- Höhenfunktion und Depolarisation, abhängig von $\Delta \mathrm{h}$

Grundlage der Rechnungen war die Einhaltung der bekannten Kurven der Schutzabstände am Rande der Versorgungsgebiete, vgl. auch Großkopf (1952). Anfangs wurde mit der Sicherung einer interferenzfreien Versorgung zu $99 \%$ der Zeit gearbeitet, d.h. störende Sender mußten durch 1\%Feldstärken berücksichtigt werden. Später setzten sich $10 \%$ für die störenden Sender durch, das entsprach dann einem interferenzfreien Empfang zu 90\% der Zeit. Mit 1\% wurde aber dann in den Fällen gerechnet, wenn es sich um internationale Abstimmungen handelt. Daraus wird deutlich, wie wichtig die Ausbreitungskurven für $10 \%$ und $1 \%$ der Zeit waren. Dass darüber hinaus markante jahreszeitliche Gänge auftreten, konnte im UKW-Rundfunkbereich, damals $87,5 \ldots 100 \mathrm{MHz}$, nach 5-jährigen Messungen im Zeitraum $1956 . .1960$ gezeigt werden, wobei 14 Sender in verschiedenen Entfernungen beobachtet wurden, eine Arbeit an der der Mitarbeiter des Verfassers W. Heide maßgeblich mitgewirkt hat.

Abbildung 1 zeigt den Turm auf dem Kolberg an dem die Meßantennen montiert waren. In Abb. 2 ist die Lage der Strecken wiedergegeben an denen mehrjährige Registrierungen bei höheren Frequenzen erfolgten, siehe auch Abb. 3 und 4 bzw. vgl. Kühn und Derer (1971), Kühn (1965).

Ein gemeinsamer Versuch wurde zwischen der ost- und westdeutschen Seite durchgeführt, obwohl es keine diesbezüglichen Absprachen gegeben hatte. An der Station Torfhaus wurde zur Bestimmung der optimalen Höhe die Antenne in verschiedene Höhen gebracht. Gleichzeitig wurde auf 


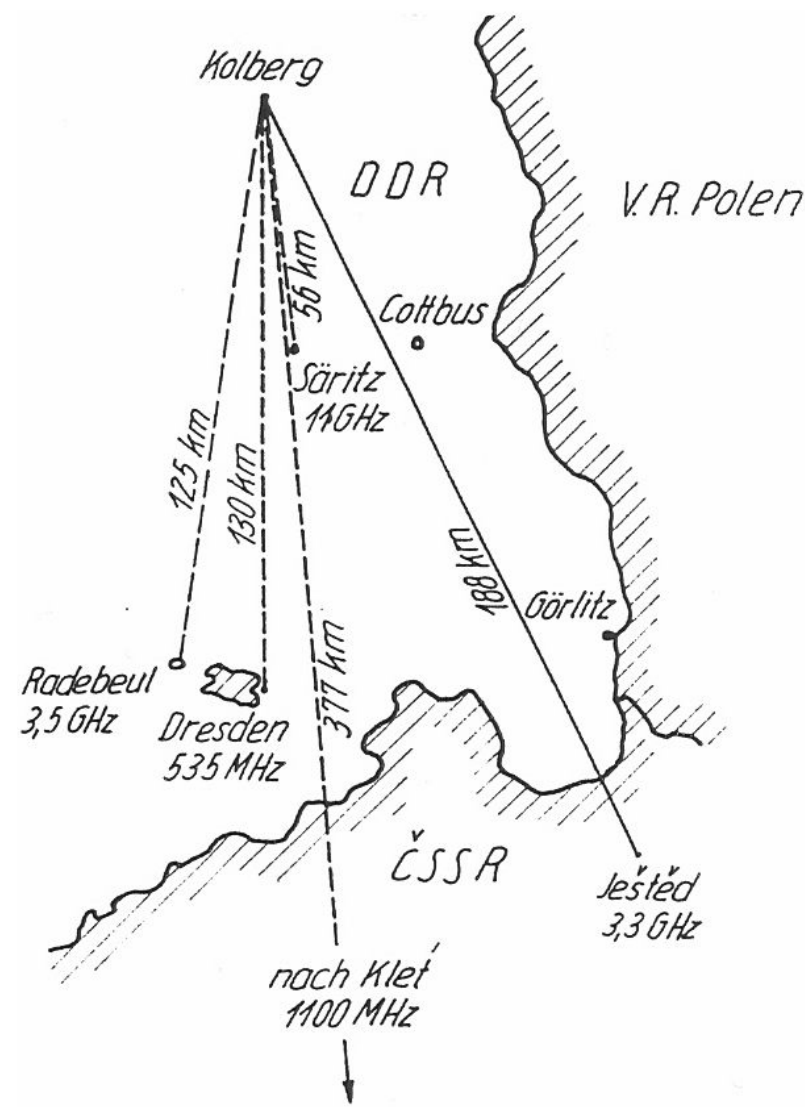

Abb. 2. Lage einiger Überhorizontstrecken an denen mehrjährige Registrierungen bei höheren Frequenzen erfolgten.

östlicher Seite in Halberstadt der Feldstärkeverlauf gemessen, wobei der Einfluß der beugenden Kante des Brocken erfaßt werden konnte. Das Ergebnis ist in Abb. 5 wiedergegeben.

Wie aus dem Vergleich mit dem theoretisch bestimmten Verlauf hervorgeht, sind die gemessenen Werte günstiger als die theoretischen.

Ein spezielles Ergebnis der troposphärischen Ausbreitungsregistrierungen war das Auftreten von auffälligen Feldstärkeanstiegen, deren Ursache zunächst unklar war. Bald wurde entdeckt, dass es sich um Effekte während des Auftretens von Gewittern handelte, welche im UKW-Bereich und bei höheren Frequenzen, bis $11 \mathrm{GHz}$ untersucht wurden mit dem Ergebnis einer ausgeprägten Frequenz- und Entfernungsabhängigkeit. Besonders wurden diese Effekte an den in Abb. 6 wiedergegebenen Strecken untersucht.

Abbildungen 7 und 8 zeigen zwei Beispiele von Gewittereffekten während der Mittagsstunden, es handelt sich also in keinem Falle um den Einfluß einer Absink- oder Strahlungsinversion.

Ein weiteres Beispiel markanter Anstiege während eines schweren Gewitters zeigt Abb. 9 für die Frequenzen $1.100 \mathrm{MHz}$ und $3.500 \mathrm{MHz}$.
Anläßlich solcher Ereignisse lassen sich die Anstiege auch bei erheblich niedrigeren Frequenzen nachweisen und können dabei innerhalb der Fernsehnetze zu Interferenzstörungen führen, die allerdings nicht von langer Dauer sind. Aus Abb. 10 ist diese Frequenzabhängigkeit deutlich erkennbar, vgl. auch Kühn (1971), Kühn (1964a).

\section{Entfernungsabhängigkeit der zeitlichen Streuung}

Wie bereits erwähnt, wurden 14 UKW-Hörfunksender über 5 Jahre gemessen und zwar im Entfernungsbereich zwischen $74 \ldots 436 \mathrm{~km}$. Beispiele für den dabei festgestellten jahreszeitlichen Gang zeigt auszugsweise für die Sender Leipzig, Hohermeißner und AFN Feldberg die Abb. 11.

Der mittlere jahreszeitliche Gang ist deutlich ausgeprägt und zeigt maximale Werte im Herbst, d.h. z.B. dei den zu 99\% freizuhaltenden Empfangsmöglichkeiten ist im Herbst mit gewissen Einschränkungen des Versorgungsgebietes zu rechnen. Weitere Hinweise finden sich in Kühn und Heide (1961).

\section{Internationale Abstimmungen}

Es erwies sich sehr schnell, dass eine nur innerdeutsch geführte Abstimmung keinesfalls ausreichend sein würde, vielmehr mußten alle angrenzenden Länder einbezogen werden. Auf östlicher Seite bedeutete dies, dass das Planungsverfahren allgemein bekannt gemacht werden mußte, und in diesem Zusammenhang ist erneut auf die Aktivitäten des Herrn Minister Probst und auf die Unterstützung durch die in Prag befindliche östliche Rundfunkunion OIR, später OIRT, hinzuweisen. So wurden in der Folgezeit vor allem in den Ländern Polen, ČSSR, Ungarn, Rumänien und UdSSR Vorträge gehalten vom Verfasser und unter maßgeblicher Beteiligung von E. Augustin, der seinerzeit technischer Leiter des RFZ war, bevor H. Stier all diese Arbeiten maßgeblich unterstützte und vorantrieb. Ihm ist es in hohem Maße zu danken, dass letztlich diese planungstechnischen Arbeiten gemeinsam mit den Untersuchungen zur Wellenausbreitung erfolgreich fortgeführt werden konnten.

Den Initiativen aus Kolberg ist es dann zuzuschreiben, dass auch eine Zusammenarbeit mit S. Ogulewicz, später A. Kawecki, vom Institut für Nachrichtentechnik I $€$ aus Warschau zustande kam.

Zur Zeit der Frequenzplanung und in den Folgejahren ergab sich mit Polen eine aktive Zusammenarbeit mit folgenden Inhalten:

- Messungen zur troposphärischen Fernausbreitung über der Ostsee bis nach Danzig sowie auf einer LandSeestrecke

- Messungen auf einer reinen Landstrecke zwischen Posen und Kolberg, $d=213 \mathrm{~km}$ 


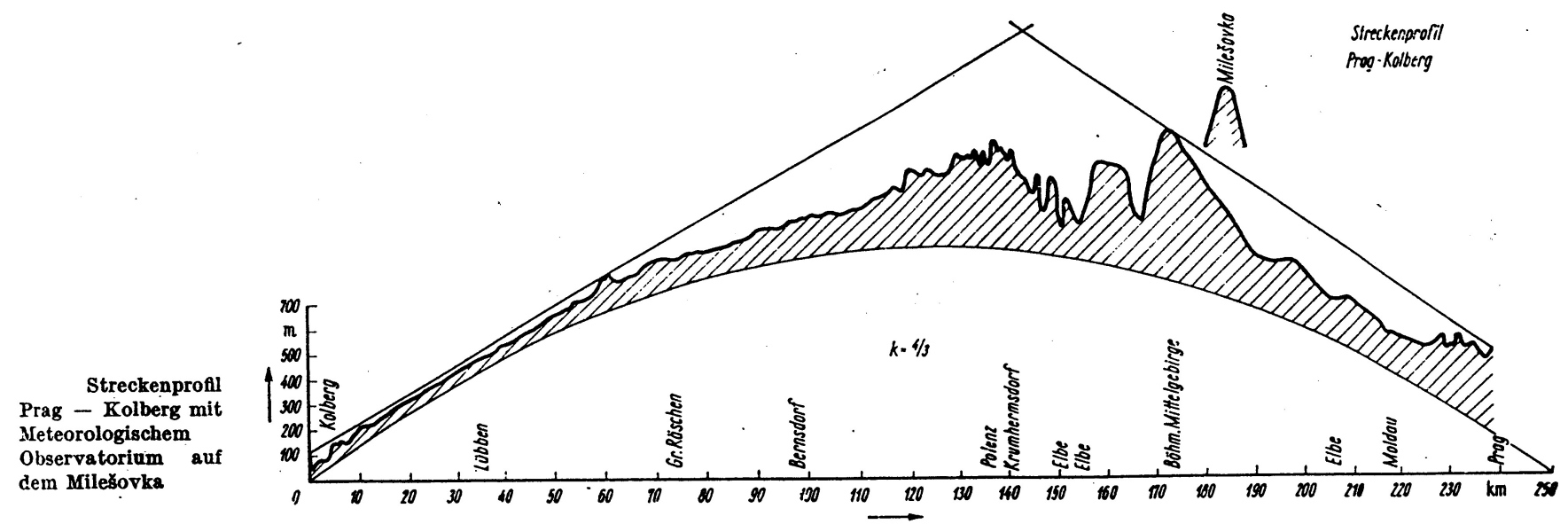

Abb. 3. Profil der Scatterstrecke Prag-Kolberg, gemeinsam betrieben mit Kollegen des VUS Prag, installiert mit P. Beckmann.

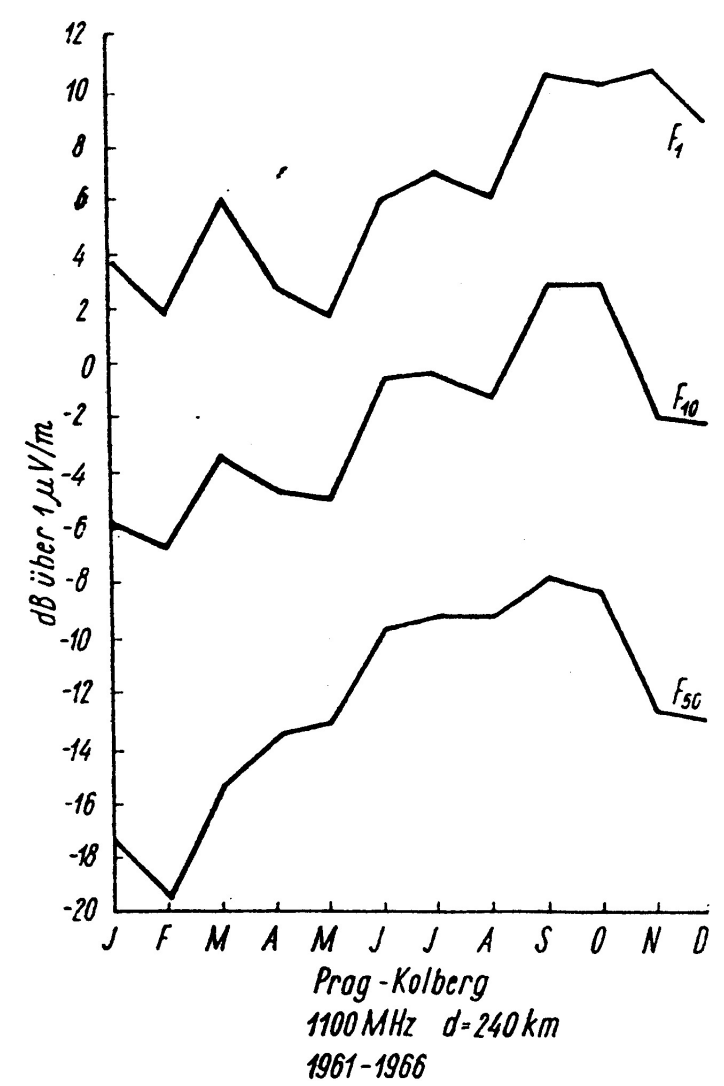

Abb. 4. Jahresgang der Feldstärke nach 6-jährigen Registrierungen an der $1.100 \mathrm{MHz}-$ Strecke Prag-Kolberg.

In ähnlicher Weise wurde eine Kooperation mit der damaligen ČSSR begonnen, wobei auf vielfältigen Gebieten zusammen gearbeitet wurde, so z.B. mit P. Beckmann und J. Pokorny bei Einrichtung und Betrieb einer Scatterstrecke zwischen Prag und Kolberg, mit J. Derer beim Betrieb einer $377 \mathrm{~km}$ langen $1100 \mathrm{MHz}$-Verbindung zwischen dem Ber- ge Klét und Kolberg. Umfangreiche Messungen erfolgten im unregelmäßigen Gelände und hinter beugenden Kanten, wobei J. Králik und V. Vočaldo aktiv mitarbeiteten. Dabei wurden die Untersuchungen konzentriert auf

- troposphärische Fernausbreitung bei 1.100 und $3.500 \mathrm{MHz}$

- Messungen im unregelmäßigen Gelände zur Ermittlung der Abweichung von den CCIR-Kurven

- Registrierungen in Fahrzeugen zur Bestimmung der Ortsstreuung

- Messungen von Depolarisation und Höhenabhängigkeit

Viele Ergebnisse wurden publiziert oder in CCIR Dokumenten zugänglich gemacht, siehe u.a. Arbeiten in Kühn (1964b), Kühn und Vočaldo (1972), Kühn (1958a), Králik et al. (1961), Ogulewicz et al. (1973).

Hauptziel war die Verifizierung der für die Planung benutzten Daten, bzw. Korrektur der verwendeten Ausbreitungskurven sowie der örtlichen Feldstärkestreuung, vor allem die Angaben über die zeitliche Streuung im Fernfeld zu verbessern.

Als wichtigste aus dieser internationalen Arbeit entstandenen Diagramme sind diejenigen über die Zeitstreuung $\mathrm{F}_{1} \ldots \mathrm{F}_{50}$ sowie die Ortsstreuung anzusehen, die in den Abb. 12 und 13 wiedergegeben sind.

Bemerkenswert ist, dass, wie noch gezeigt wird, die Entfernungsabhängigkeit der zeitlichen Streuung mit zunehmender Frequenz noch stärker ausgeprägt ist. Auch die örtliche Streuung ist stark frequenzabhängig. Daneben findet sich ein dominierender Einfluß der Geländegestaltung, hier beschrieben durch den $\Delta \mathrm{h}$-Wert. Diese Definition hat sich allgemein eingeführt und wird zur Geländebeschreibung weitgehend benutzt, obgleich die Hangneigung vernachlässigt wird, was sich aber nicht als hinderlich erwiesen hat. 


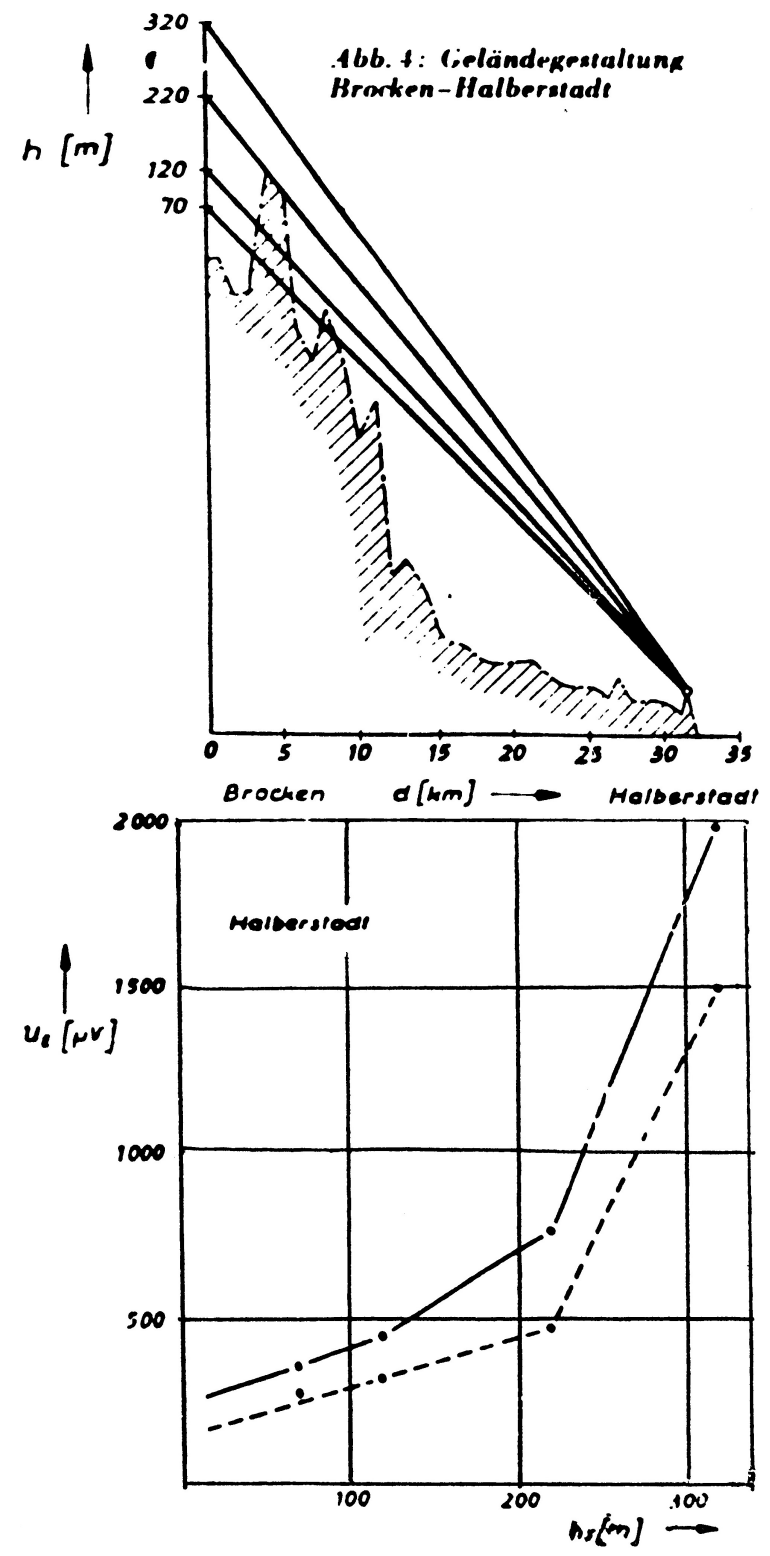

Abb. 5. Messung im UKW-Bereich hinter einer beugenden Kante bei verschiedenen Sendeantennenhöhen.

Da die Registrierung der Ortsstreuung an einer Vielzahl von Sendern erfolgte, konnte umfangreiches Material aus allen Teilen des Landes ausgewertet werden. Im Ergebnis wird es möglich, eine Karte der regionalen Verteilung dieses Wertes zu entwerfen. Für die Frequenzen $480 \mathrm{MHz}$ und $780 \mathrm{MHz}$ sind die entsprechenden regionalen Verteilungen in den Abb. 14 und 15 wiedergegeben.

Ein Vergleich der Entfernungsabhängigkeit der Medianwerte mit der CCIR-Kurve ist in Abb. 16 gezeigt.

Um eine Vergleichbarkeit zu erreichen, und da die Antennen über unterschiedliche Höhen verfügten, wurde eine Höhenkorrektur vorgenommen. Wie aus Abb. 16 ersichtlich, liegen alle $\mathrm{F}_{50}$-Werte auf oder unterhalb der CCIR-Kurve

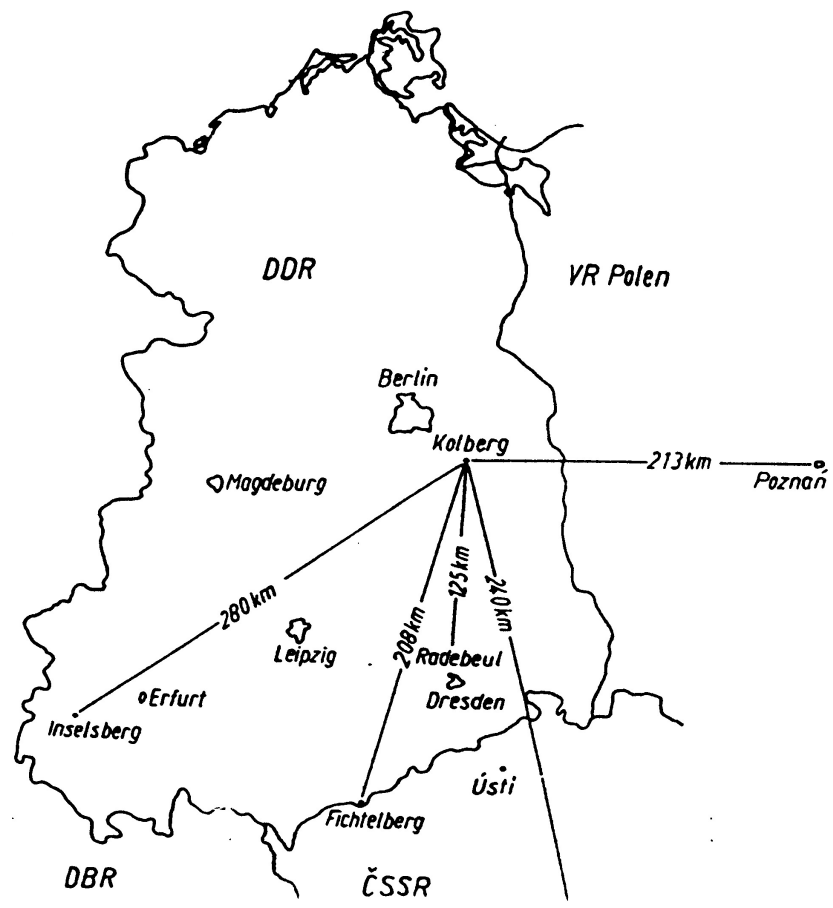

Abb. 6. Lage der Meßstrecken zur Untersuchung der Entfernungsabhängigkeit des Gewittereffektes.
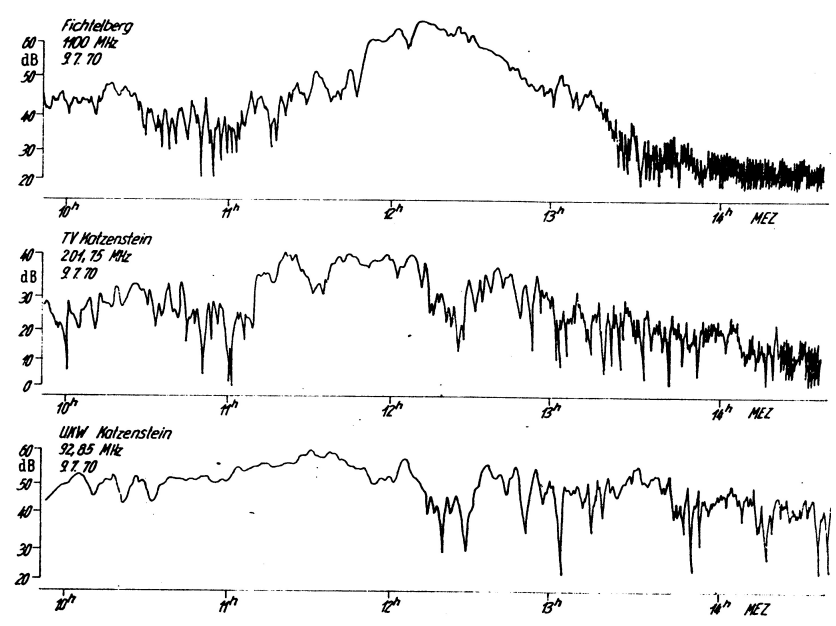

Abb. 7. Feldstärkeanstiege durch Gewitter am 9.Juli 1970 bei nahbenachbartstehenden Sendern, Empfangsstelle Kolberg.

gültig für den UHF Bereich, also Band 4/5. Die Feldstärken von Fichtelberg sind deutlich höher und diejenigen von Prag wesentlich tiefer, was sicher als Folge der sehr unterschiedlichen Streckengestaltung zu deuten ist.

Die in Kolberg gemessene Kurve für Band 1 bis 3 stimmt recht gut mit derjenigen des CCIR überein. Besonders deutlich ist die Abweichung der zeitlichen Streuung von den CCIR-Daten erkennbar, wie Abb. 17 zeigt.

Dass der bei Prag gemessene Wert so niedrig ist, hängt eindeutig mit dem Streckenprofil zusammen. Es wird das 


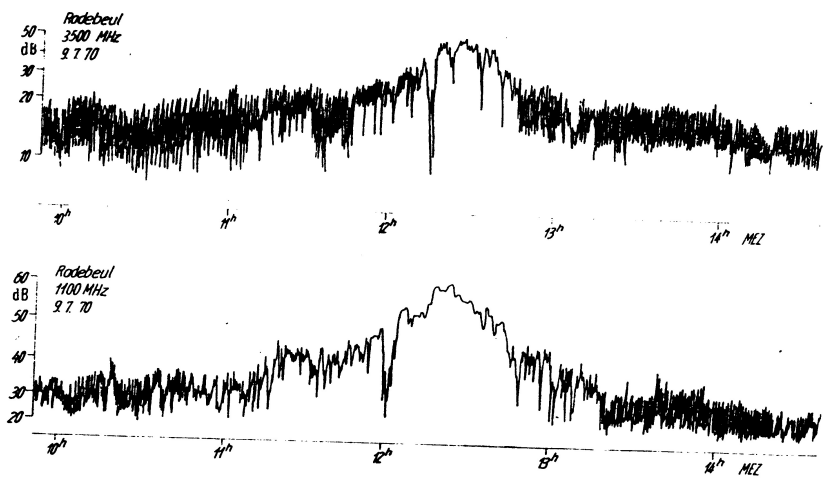

Abb. 8. Gewittereffekt bei $1.100 \mathrm{MHz}$ und $3.500 \mathrm{MHz}$ am 9. Juli 1970 an der 125 km-Strecke Radebeul-Kolberg.

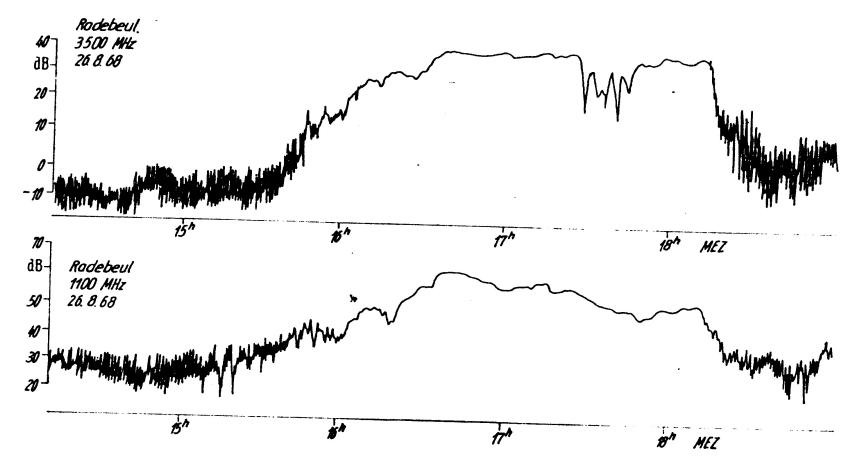

Abb. 9. Feldstärkeanstiege während eines schweren Gewitters an der Strecke Radebeul-Kolberg, d=125 km am 26. August 1968.

Böhmische Mittelgebirge und Teile des Erzgebirges überquert, wobei sich die Strecke als ausgeprägte Scatterverbindung erweist an der auch keine markanten Feldstärkeanstiege bei Inversionen auftraten, auch gab es keine Gewittereffekte, somit bleibt die zeitliche Streuung bei so niedrigen Werten, wie in der Abbildung dargestellt.

In ungefährer Höhe des Scattervolumens befindet sich auf dem Berge Milešovka eine meteorologische Station. So ergab sich die Möglichkeit, aus den dort gemessenen Daten die Monatswerte des Brechungsindex zu bestimmen und diesen mit den über 7 Jahre registrierten $\mathrm{F}_{50}$-Werten der Feldstärke $\mathrm{zu}$ vergleichen, wie Abb. 18 zeigt. Die Daten erschienen vor allem von Interesse, da es in Mitteleuropa so langdauernde Meßreihen allgemein nicht gibt.

Auf eine Besonderheit ist noch hinzuweisen. Die $377 \mathrm{~km}$ lange Verbindung Klét-Kolberg verläuft zu großen Teilen über das Böhmische Becken und zeigt wegen der großen Entfernung das Verhalten einer Scatterverbindung und Feldstärken erheblich geringere Werte verglichen mit den UHF-Kurven des CCIR, jedoch kommt es für geringe Zeitprozente zu erheblichen Feldstärkeanstiegen, wie die Abb. 19 erkennen läßt. Ursache dafür sind offensichtlich Inversionen, welche bevorzugt im Herbst auftreten. Die effektive Antennenhöhe am Berge Klét beträgt $400 \mathrm{~m}$.

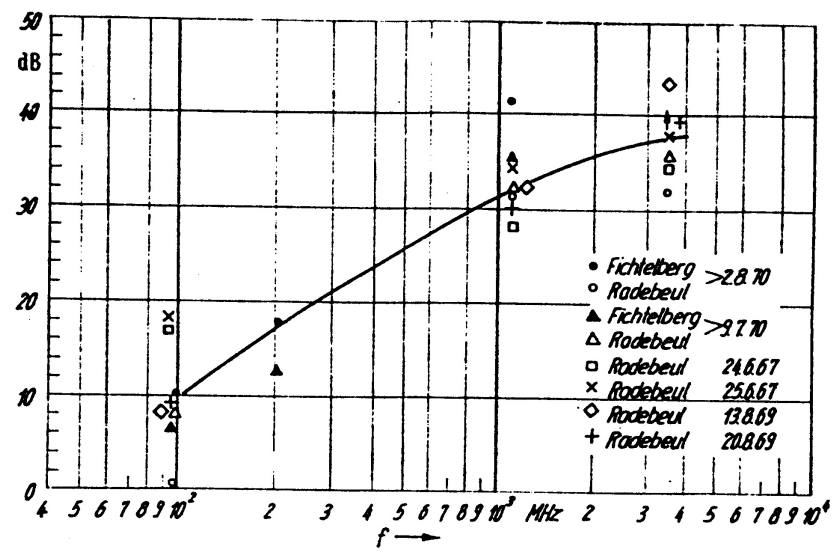

Abb. 10. Frequenzabhängigkeit von Feldstärkeanstiegen bei Gewittern. Die Abszisse zeigt die Frequenz, die Ordinate den Betrag des Anstiegs in dB.

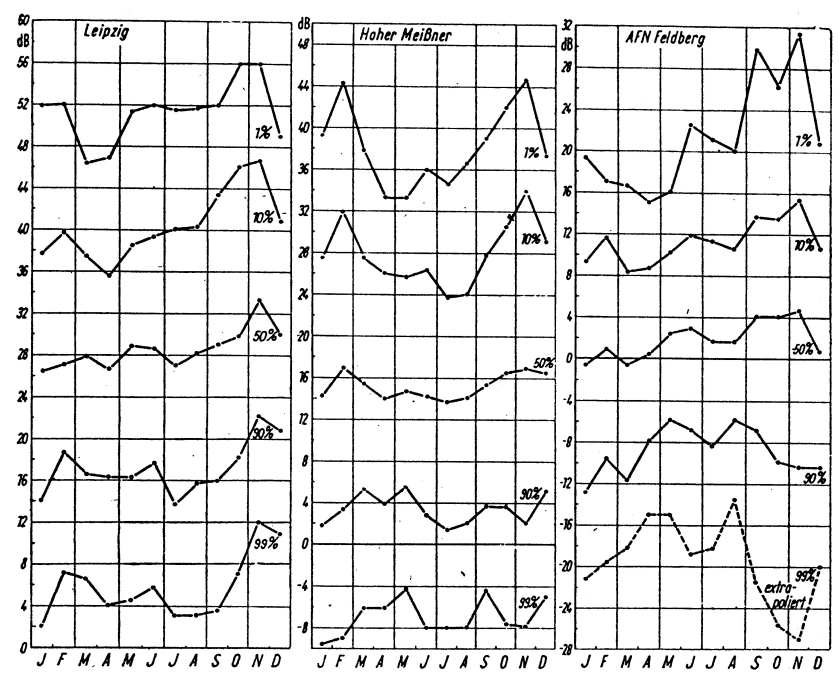

Abb. 11. Jahresgang der $F_{1}, F_{10}, F_{50}, F_{90}$ und $F_{99}$ Werte nach 5jährigen Messungen der UKW-Hörrundfunk-Sender Leipzig, $d=$ $140 \mathrm{~km}$, Hoher Meißner, $\mathrm{d}=295 \mathrm{~km}$ und AFN-Feldberg, $\mathrm{d}=$ $436 \mathrm{~km}$.

\section{Ausbreitung über der Ostsee}

Da in dem mitteleuropäischen Raum auch Sender aus nördlichen Richtungen bei den Interferenzbetrachtungen berücksichtigt werden müssen, erfolgten gemeinsam Messungen mit den Kollegen in Warschau, vor allem mit S. Ogulewicz. Anfangs wurde nur die Verbindung Jaroslawiec-Saßnitz im UHF Bereich betrieben, die ausschließlich über See verlief. Die später eingerichtete Strecke Szczecinek-Saßnitz war eine Land-Seestrecke und die Verbindung nach Ückermünde lief ausschließlich über Land, wie Abb. 20 erkennen läßt.

Da diese Untersuchungen über mehrere Jahre erfolgen mußten, ließ das Interesse der wissenschaftlichen Leitung des RFZ bald erheblich nach, und es waren erhebliche 


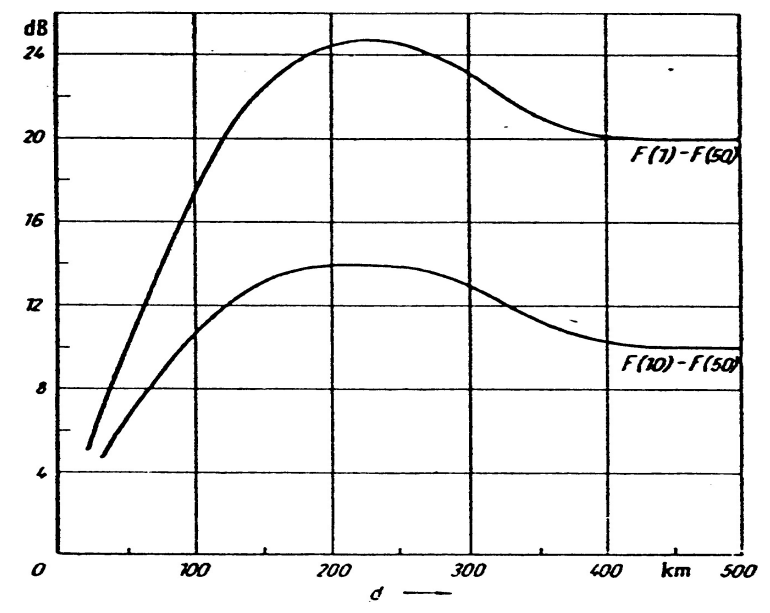

Abb. 12. Entfernungsabhängigkeit der zeitlichen Streuung im UKW-Hörrundfunkband nach 5-jährigen Messungen in Kolberg.

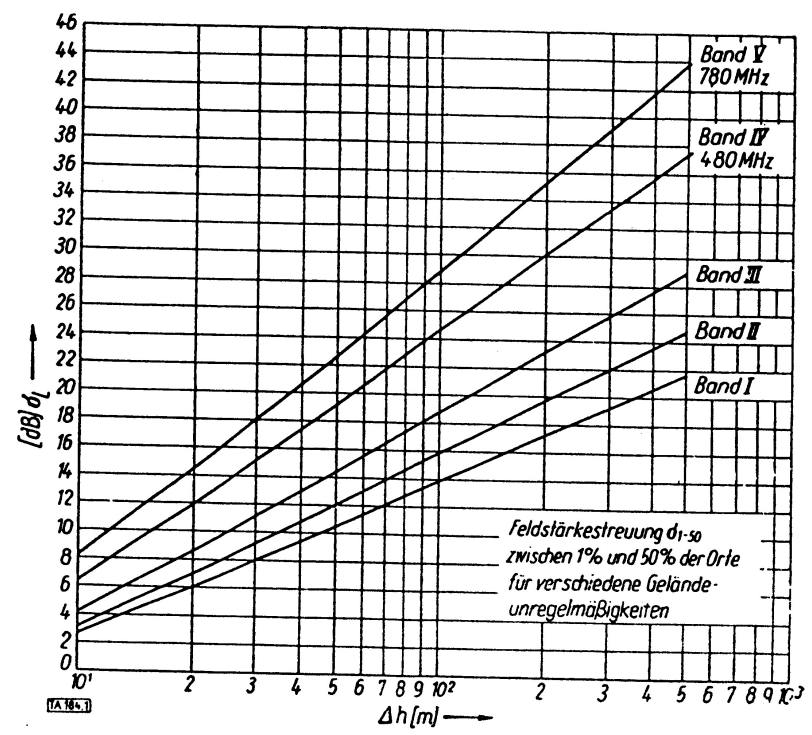

Abb. 13. Diagramm zur Ermittlung der örtlichen Feldstärkestreuung bei den verschiedenen Frequenzbändern in Abhängigkeit von der Geländeunregelmäßigkeit $\Delta \mathrm{h}$.

Anstrengungen notwendig um die Arbeiten zu einem erfolgreichen Abschluß zu bringen, z.B. Kühn et al. (1973a), Kühn und Ogulewicz (1970), Kühn et al. (1973b), Ogulewicz et al. (1976).

Später wurden die Entfernungen vergrößert, bis über $300 \mathrm{~km}$, Abb. 21.

Der innerhalb von 3 Jahren gemessene Verlauf der $\mathrm{F}_{50}$, $F_{10}, F_{5}$ und $F_{1}$-Werte ist in Abb. 22 gezeigt. Der jahreszeitliche Gang der Einzelwerte und damit der zeitlichen Streuung ist erheblich, und die Maximalwerte werden vornehmlich an der reinen Seestrecke im Sommer erreicht.

Der mittlere Jahresgang an der gleichen Strecke ist in der Abb. 23 dargestellt.

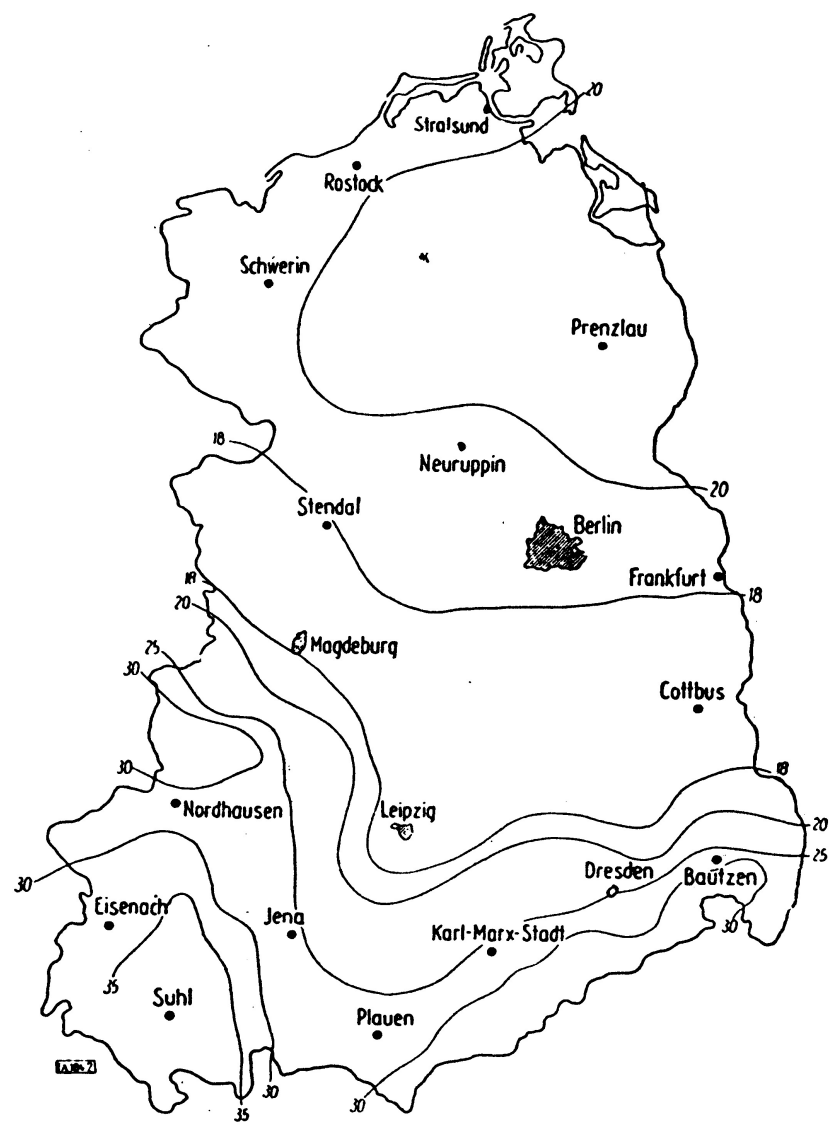

Abb. 14. Regionale Verteilung der örtlichen Feldstärkestreuung $\mathrm{F}_{1,50} \ldots \mathrm{F}_{50,50}$ bei $480 \mathrm{MHz}$.

Vergleichsweise zeigt Abb. 24 den mittleren Jahresgang ebenfalls nach 3-jährigen Registrierungen an der $221 \mathrm{~km}$ Land-Seestrecke Sczcecinek-Saßnitz.

An der Land-Seestrecke ist nicht nur der Jahresgang geringer ausgeprägt, es ist auch das Maximum deutlich auf den Herbst verschoben. Während tageszeitliche Gänge auf Strecken über Land wegen der nächtlichen Inversionsbildung stark ausgeprägt sind, ist die Variation während eines Tages über See kaum erkennbar. Abbildung 25 zeigt dies für die reine Seestrecke mit $\mathrm{d}=189 \mathrm{~km}$ sehr deutlich.

Dass interferierende Sender bei Ausbreitung über Wasser hohe zeitliche Streuungen hervorbringen, läßt sich aus Abb. 26 leicht erkennen. Diese zeigt die mittlere zeitliche Streuung und nach dreijährigen Registrierungen mit den während Mai bis August nahezu gleich hohen Werten für $\mathrm{F}_{1} \ldots \mathrm{F}_{50}$ von über $50 \mathrm{~dB}$.

Auf Initiative der polnischen Seite wurde die Entfernung auf über $300 \mathrm{~km}$ ausgedehnt und zwar auf die reine Seestrecke Rozewie-Saßnitz und die nahezu gleich lange Verbindung Danzig-Saßnitz, wie in Abb. 21 dargestellt. Wegen der großen Entfernungen war es erforderlich, auch aufwendigere Antennen einzusetzen, wie beispielsweise Abb. 27 für die Station Rozewie zeigt, wo die Antenne auf dem dortigen 


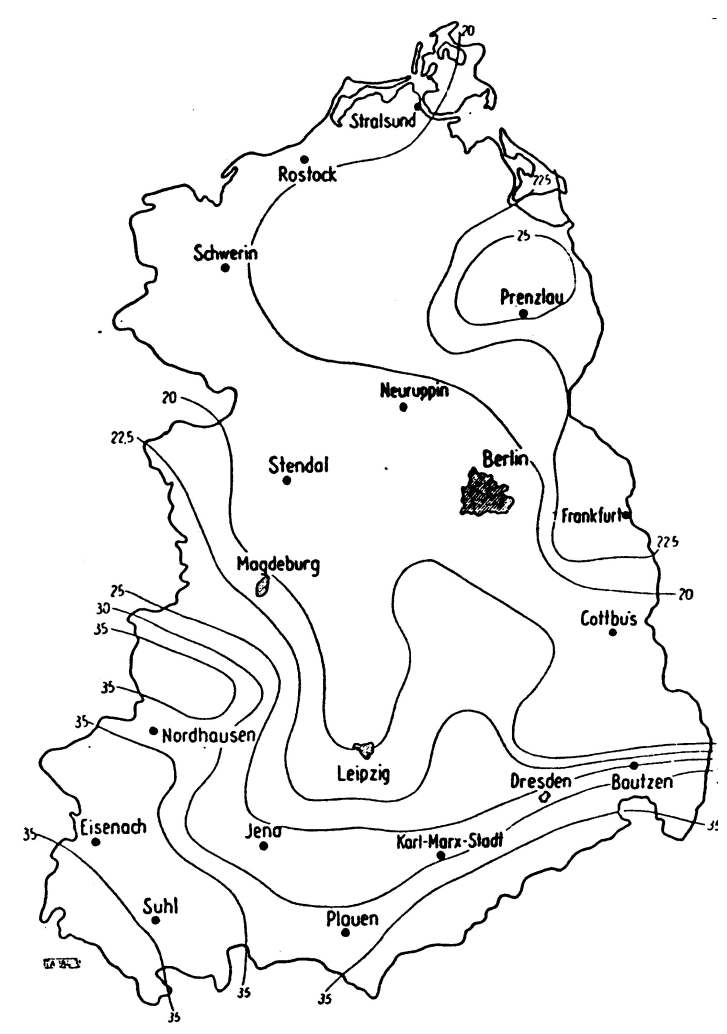

Abb. 15. Regionale Verteilung der örtlichen Feldstärkestreuung $\mathrm{F}_{1,50} \ldots \mathrm{F}_{50,50}$ bei $780 \mathrm{MHz}$.

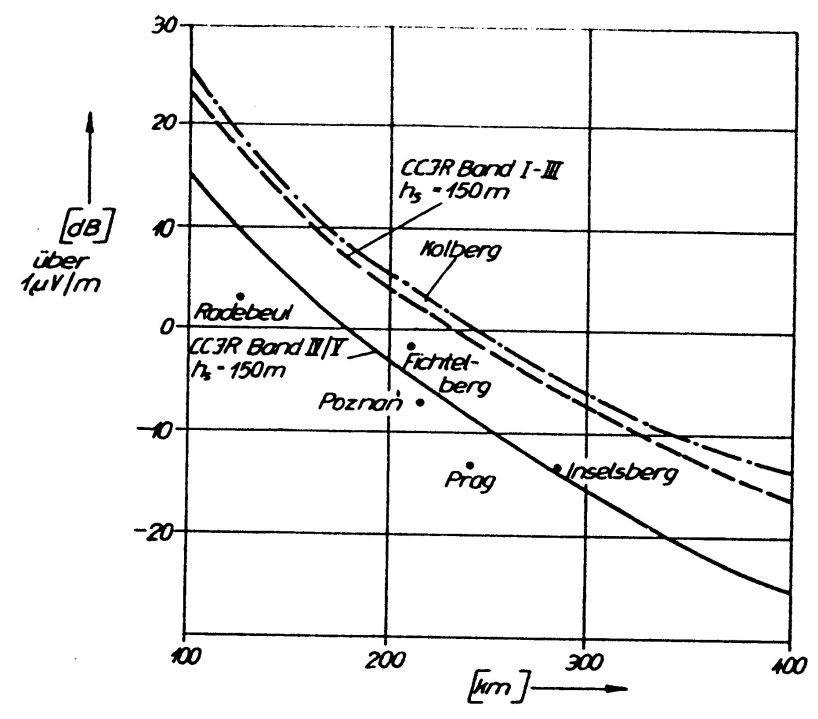

mit Hohenkorrehturen

Abb. 16. Ergebnisse 3-jähriger Messungen bei $1.100 \mathrm{MHz}$ mit CCIR-Kurve Band 1 bis 3 und $h_{s}=150 \mathrm{~m}$ sowie derjenigen der Bänder 4/5.

Leuchturm montiert war. Die Meßwerte an dieser nahezu reinen Seestrecke mit nur 11\% Landteil zeigt Abb. 28.

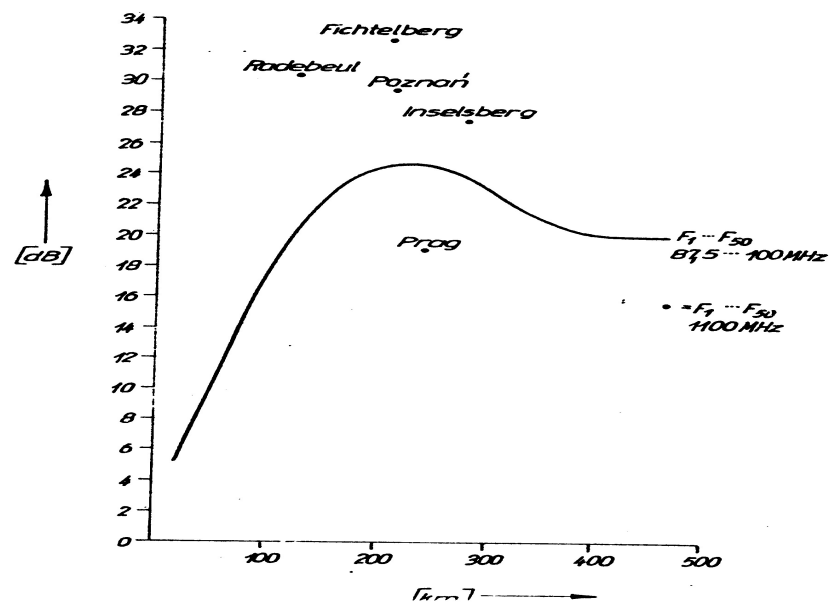

Abb. 17. Verlauf der zeitlichen Streuung im UKW-Rundfunk-Band $87,5 \ldots 100 \mathrm{MHz}$, ausgezogene Kurve, und zeitliche Streuung bei 1.100 MHz der Sender Radebeul, Fichtelberg, Prag und Inselsberg sowie Poznan.

Beachtliche zeitliche Streuungen treten auch bei so großen Entfernungen noch auf, wie z.B. im Sommer 1972 als im Juli $\mathrm{F}_{1} \ldots \mathrm{F}_{50}=50 \mathrm{~dB}$ erreicht wurden.

Zur besseren Übersicht wurde der im gesamten Meßzeitraum beobachtete mittlere jahreszeitliche Gang in Abb. 29 dargestellt. In 8 Monaten ließ sich der $\mathrm{F}_{50}$-Wert nicht ermitteln. Für die $F_{10}, F_{5}$ und $F_{1}$-Werte zeigte sich das Maximum im Juli.

\section{Anwendungen der Messergebnisse}

Ziel aller Untersuchungen war es, die verwendeten Parameter der Wellenausbreitung auf ihre Eignung in Mitteleuropa zu prüfen und wenn erforderlich zu korrigieren. Wo es wenig oder keine Daten gab, sollten neue geschaffen werden, wie z.B. bei der Ausbreitung über der Ostsee, weshalb der Zusammenarbeit mit Polen verstärkte Aufmerksamkeit gewidmet wurde. Als verbesserungsbedürftig erwiesen sich auch die anfangs vorhandenen Daten der Ortsstreuung. Hierbei ist nicht nur auf die Messungen innerhalb des eigenen Landes sondern auch auf vielfältige Untersuchungen in der damaligen ČSSR hinzuweisen. Alle Ergebnisse fanden ihren Niederschlag im Diagramm zur Abhängigkeit der örtlichen Feldstärkestreuung von Frequenz und Geländeunregelmäßigkeit. Ursprünglich wurde lediglich ein diskreter Wert bei der Netzplanung verwendet. Nach Vorliegen der umfangreichen neueren Ergebnisse wurde es möglich, genauer nach Frequenz und Geländestruktur zu differenzieren.

Letztlich konnten Karten der regionalen Verteilung der Ortsstreuung ermittelt werden, wie Abb. 14 und 15 zeigen.

Deutlich läßt sich aus den Verteilungen die Geländestruktur des Landes ablesen, mit stärker gegliederten Teilen im Süden und Südwesten und vorwiegend flachen Gegenden 


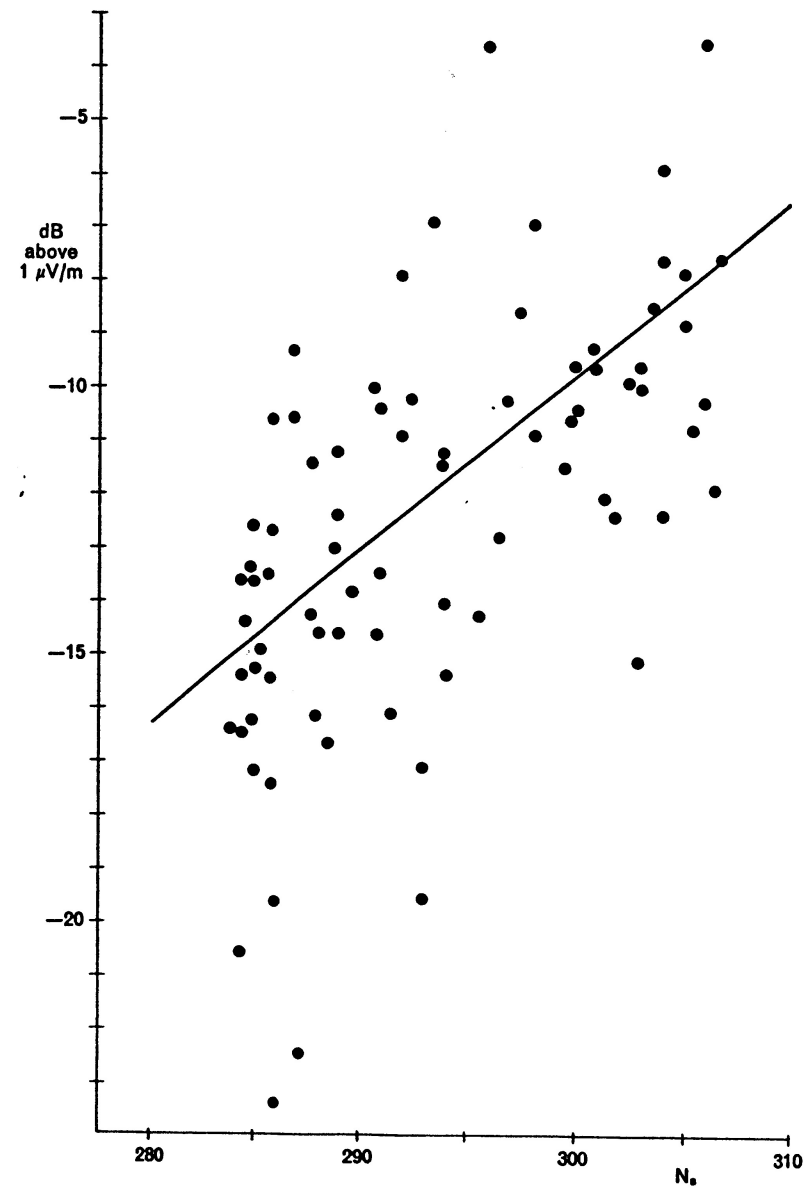

Abb. 18. Monatliche $\mathrm{F}_{50}$-Werte der Feldstärke an der $1.100 \mathrm{MHz}$ Scatterstrecke Prag-Kolberg, $d=240$ km nach 7-jährigen Registrierungen und Monatswerte der Refraktivität an der Station Milešovka, $835 \mathrm{~m}$ über NN.

im Norden. So werden im Nordosten weniger als $20 \mathrm{~dB}$ für die $\mathrm{F}_{1,50} \ldots \mathrm{F}_{50,50}$-Werte bei $480 \mathrm{MHz}$ gefunden, dagegen mehr als $30 \mathrm{~dB}$ im Süden und mehr als $35 \mathrm{~dB}$ im Südwesten, dem Gebiet des Thüringer Waldes. In der Praxis bedeutet dies kleinere Versorgungsgebiete im südlichen Landesteil und größere im Norden, verglichen mit der anfänglichen Verwendung eines einheitlichen Wertes von ca. $19 \mathrm{~dB}$. Um bei den dichten Sendernetzen in Mitteleuropa den notwendigen Schutzabstand einzuhalten, wird es mitunter erforderlich die Sender orthogonal polarisiert zu betreiben. Von dieser Möglichkeit wurde bei der Planung auch Gebrauch gemacht. Die für $90 \%$ der Orte zu erzielende Verbesserung wurde dabei mit $10 \mathrm{~dB}$ angesetzt. Da damals dieser Wert nicht ausreichend belegt erschien, wurde eine Vielzahl spezieller Messungen gemeinsam mit den Herren Králik und Vočadlo in der ČSSR angestellt. Vorbereitend dazu entwickelte mein Mitarbeiter J. Käbe eine Antenne, die in $10 \mathrm{~m}$ über Grund auf einem Meßfahrzeug montiert war und deren Polarisation vom Inneren des Meßwagens aus von einer Polarisationsart auf die andere positioniert werden konnte. Ein extra montiertes

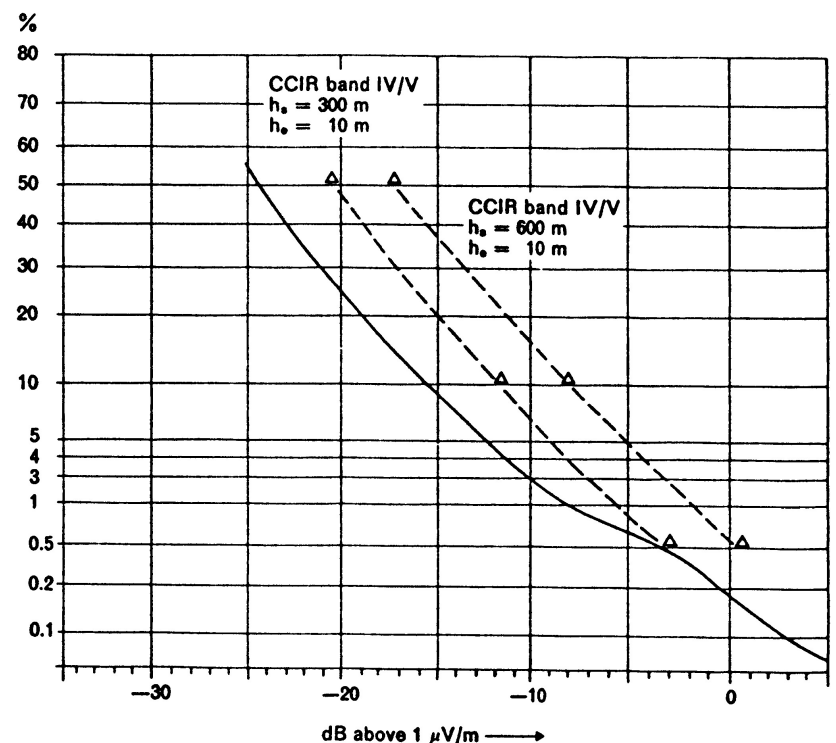

Abb. 19. Häufigkeitsverteilung der Feldstärke bei $1.100 \mathrm{MHz}$ an der Strecke Klét-Kolberg und CCIR-Kurven für Band 4/5.

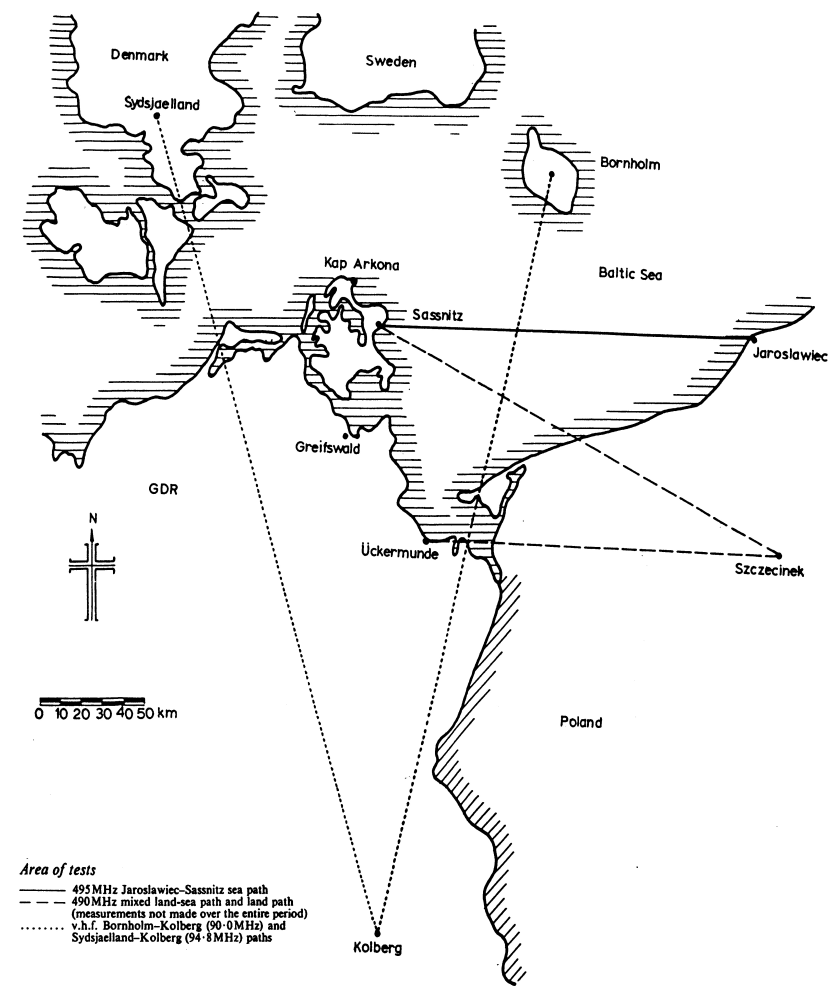

Abb. 20. Lage der Meßstrecken bei 90,0 MHz, BornholmKolberg und 94,8 MHz, Sydsjaelland-Kolberg sowie der UHFStrecken Jaroslawiec-Saßnitz, Szczecinek-Saßnitz und SzczecinekÜckermünde.

Gewicht sorgte dafür, dass auch bei schrägem Untergrund die exakte Polarisationsebene eingehalten werden konnte. Die 


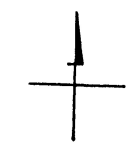

OSTSEE

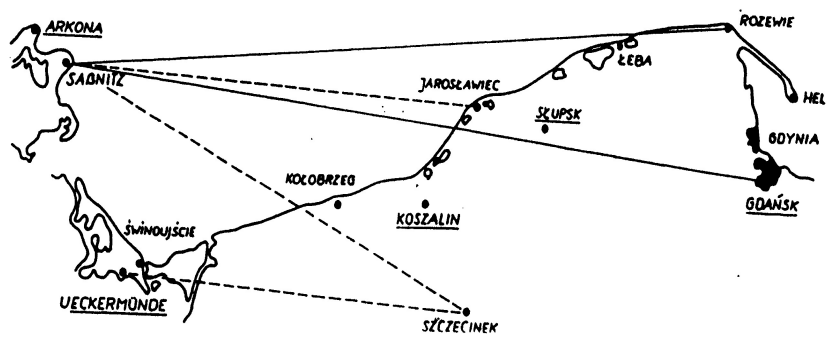

Abb. 21. UHF-Strecken über $300 \mathrm{~km}$.

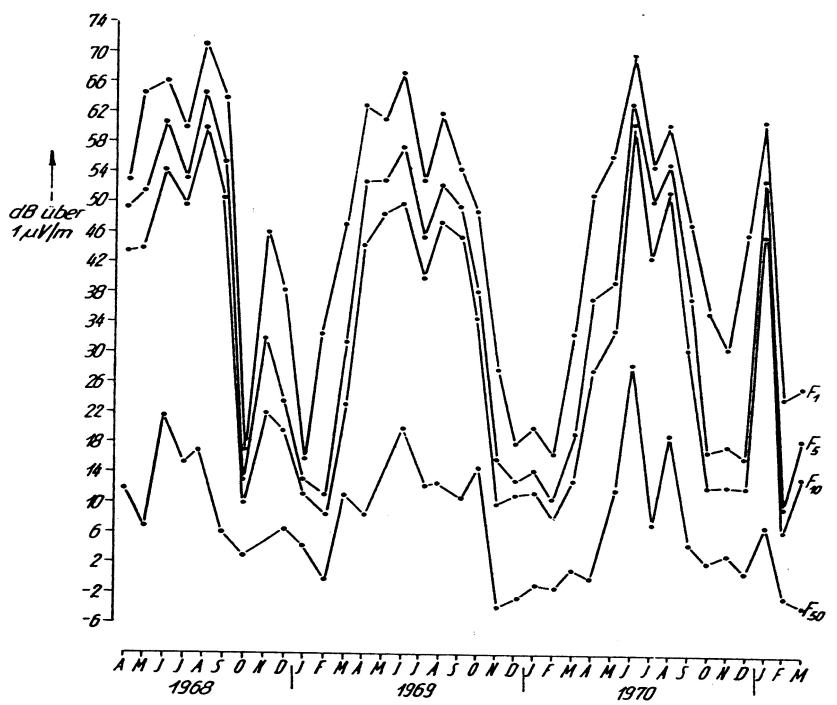

Abb. 22. Verlauf der Feldstärken an der $189 \mathrm{~km}$ langen Seestrecke Jaroslawiec-Saßnitz, 495 MHz.

Abb. 30 zeigt das Ergebnis derartiger Messungen im Stadtgebiet von Dresden, siehe auch Kühn (1962).

\section{Diskussion}

Die Frage, ob das erwartete Ergebnis der gemeinsamen Frequenzplanung letztendlich erreicht wurde, kann eindeutig mit ja beantwortet werden. Nicht nur wurde das einheitliche Planungsverfahren allgemein zur Anwendung gebracht, es konnten auch neue Wellenausbreitungsparameter zur Verbesserung der Methodik eingeführt werden. So ergaben sich neue Erkenntnisse, zur troposphärischen Fernausbreitung, verbesserte Ausbreitungskurven, vor allem im UHF-Bereich, verbesserte Daten über die örtliche Feldstärkestreuung, sowie zur Ausbreitung über See.

Die Auswirkungen für die innerdeutsche Planung lassen sich am Beispiel der Versorgungsgebiete nach OIR und nach CCIR erkennen, wie Abb. 31 und 32 zeigen (vgl. auch Kühn,

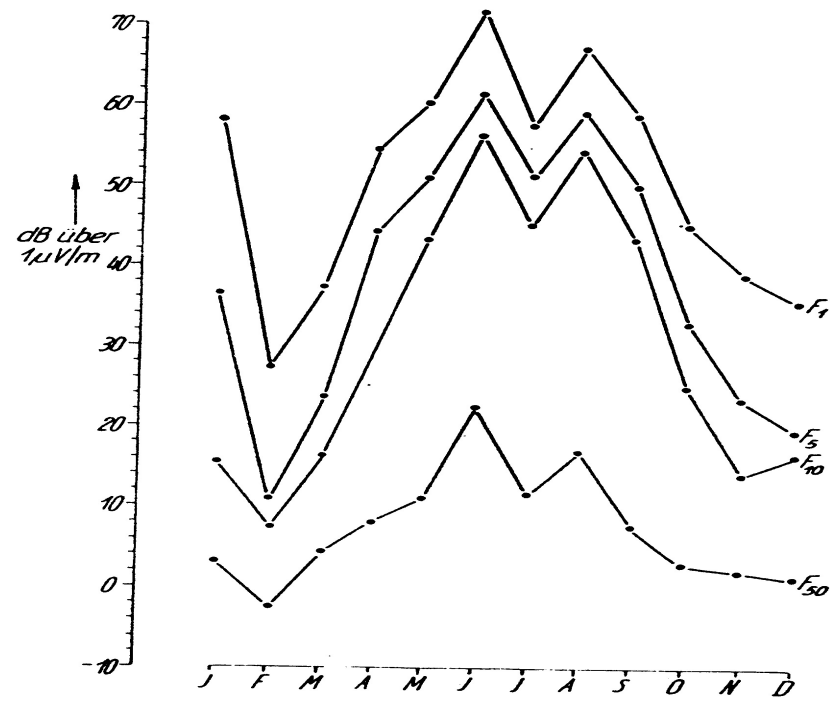

Abb. 23. Mittlerer Jahresgang nach 3-jährigen Messungen gemäß Abb. 18

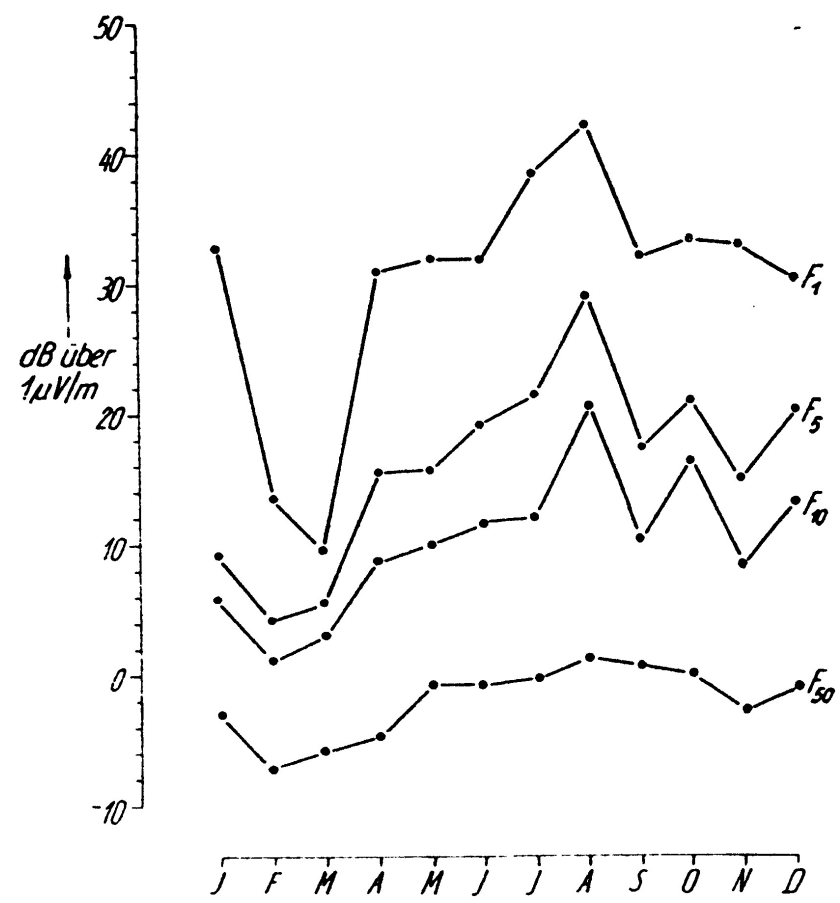

Abb. 24. Jahresgang der Feldstärken an der Land-Seestrecke Szczecinek-Saßnitz, $485 \mathrm{MHz}, \mathrm{d}=221 \mathrm{~km}$.

1958b). Bei den Karten der vorstehenden Abbildungen ist zu berücksichtigen, dass im Planungsverfahren zunächst mit Rundempfangsantennen gerechnet wurde. In der Praxis werden jedoch vorwiegend Yagi-Antennen eingesetzt, die ein besseres Signal des Nutzsenders liefern, dagegen die seitlich oder von rückwärts einfallenden interferierenden Sender mehr oder weniger abschwächen. Dadurch sind die Versorgungsflächen in der Praxis erheblich größer. Zur Schließung 

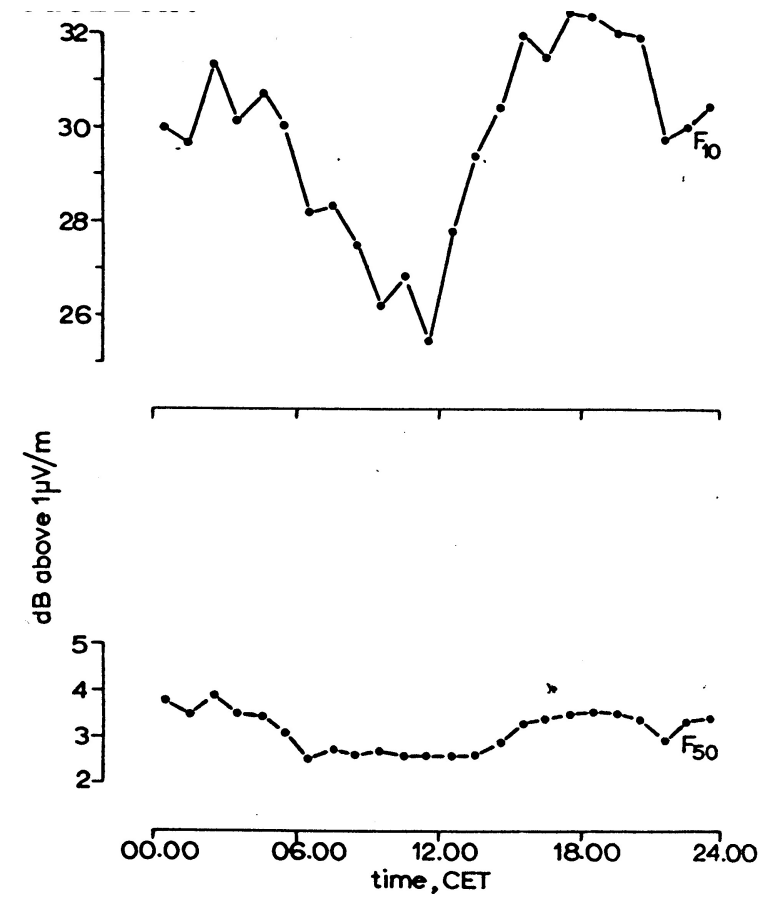

Abb. 25. Mittlerer Tagesgang der $\mathrm{F}_{50^{-}}$und $\mathrm{F}_{1}$-Feldstärken an der $189 \mathrm{~km}$ langen Seestrecke, $495 \mathrm{MHz}$.

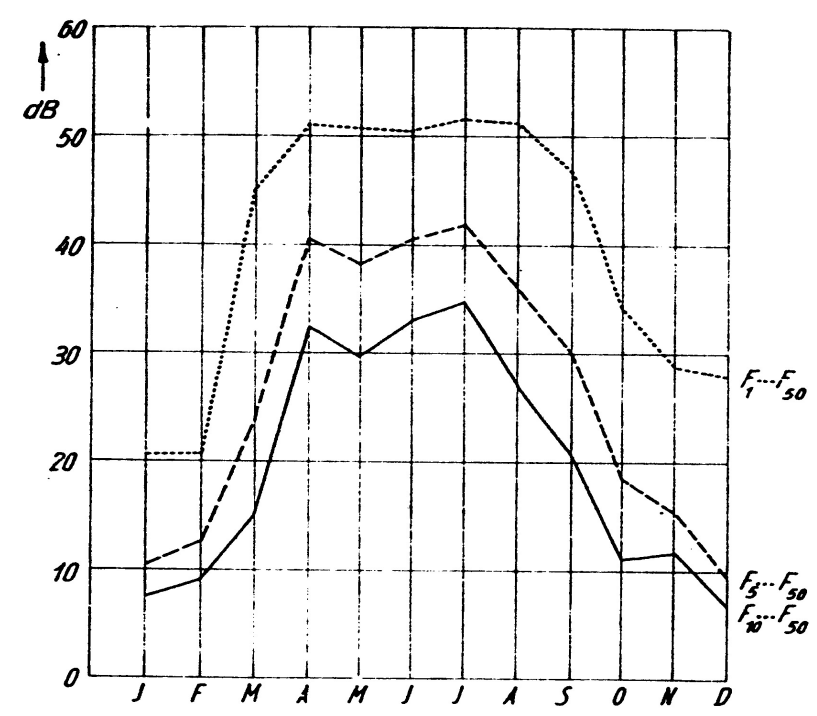

Abb. 26. Mittlerer Jahresgang der zeitlichen Streuung $F_{5} \ldots F_{50}$ und $\mathrm{F}_{10} \ldots \mathrm{F}_{50}$ auf der Strecke Jaroslawiec-Saßnitz, $495 \mathrm{MHz}$.

der Versorgungslücken sollten weitere kleinere Sender zum Einsatz kommen.

Dieser Aufsatz über die gemeinsame Planung erschien seinerzeit in der von H. Fleischer herausgegebenen Zeitschrift Fernmeldepraxis (Kühn, 1958b). Die Arbeit erregte seinerzeit bei der schwierigen politischen Situation erhebliche Aufmerksamkeit, sodass sich auch andere Fachzeitschriften zu einem kompletten Nachdruck entschlossen, wie die „Nach-

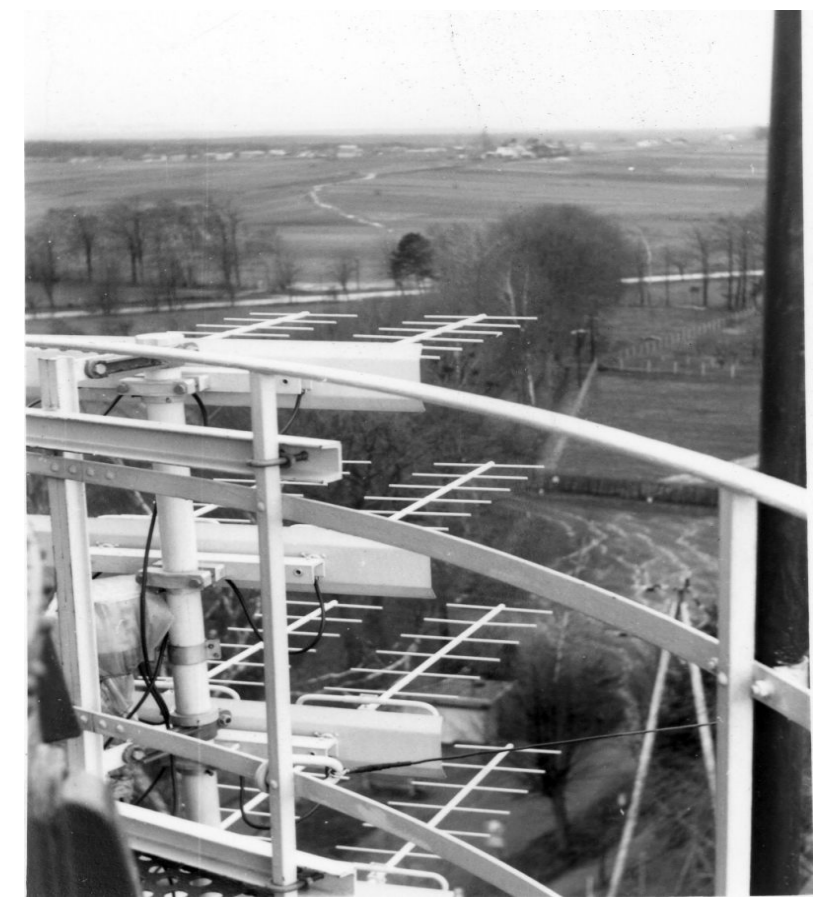

Abb. 27. UHF-Antenne am Leuchtturm Rozewie nördlich Danzig.

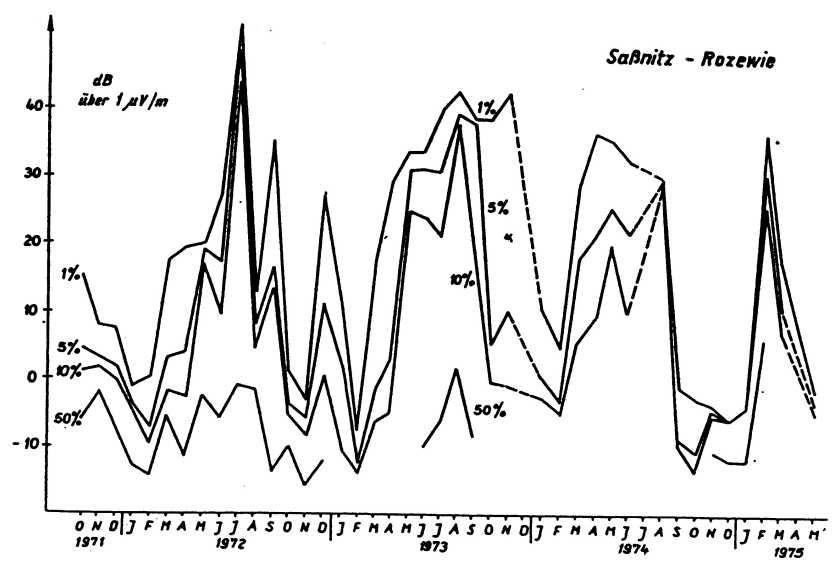

Abb. 28. Feldstärkeverlauf Saßnitz-Rozewie d = 302 km im Meßzeitraum.

richtentechnik“, „Radio und Fernsehen“ und das Publikationsorgan der Post, „Die Deutsche Post“.

\section{Anmerkung}

Abschließend möchte der Verfasser darauf aufmerksam machen, dass die gemachten Ausführungen nur einige Hinweise auf Arbeiten des FTZ/Darmstadt bringen können, da ihm nicht alle Einzelheiten und Sachverhalte bekannt sind. Die seinerzeit dort tätigen Kollegen wären viel besser in der Lage über dieses so wichtige Kapitel der Entwicklung des Hörrundfunks und Fernsehens zu berichten. Einige Namen 


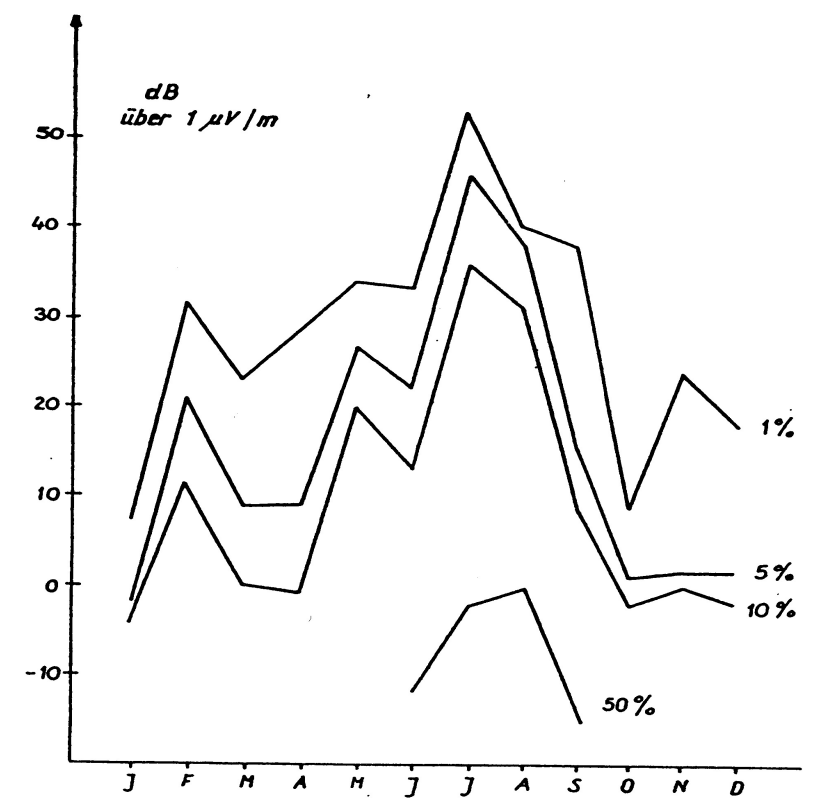

Abb. 29. Mittlerer Jahresgang der Feldstärken für 50, 10, 5 und 1\% der Zeit an der Seestrecke Saßnitz-Rozewie, $485 \mathrm{MHz}, \mathrm{d}=302 \mathrm{~km}$

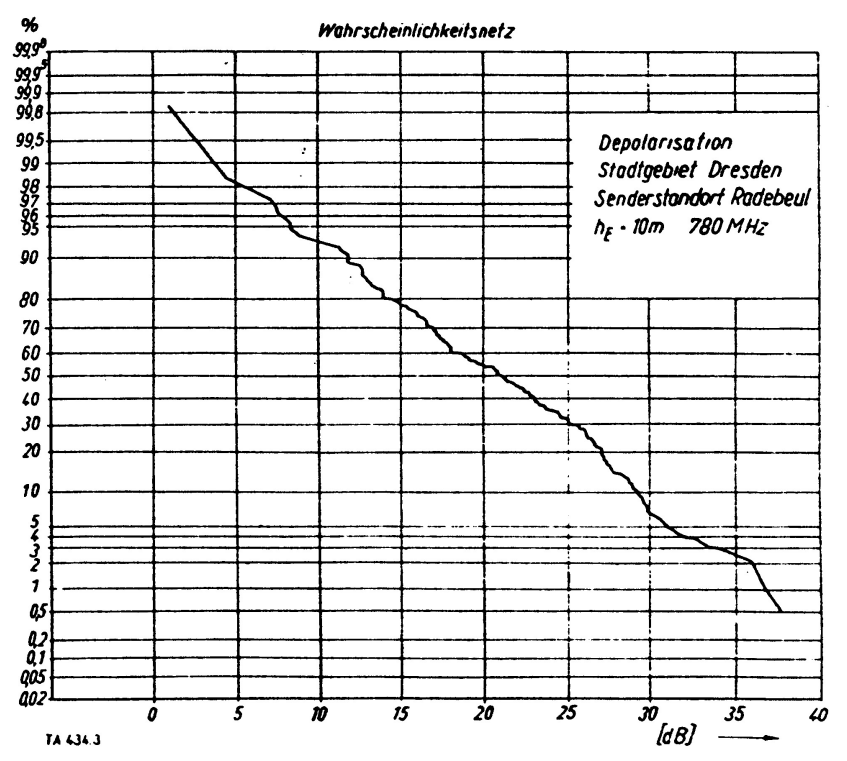

Abb. 30. Feldstärkeverhältnis zwischen horizontaler und vertikaler Polarisation bei $780 \mathrm{MHz}$ im Stadtgebiet von Dresden, $\mathrm{h}_{\mathrm{E}}=10 \mathrm{~m}$ und horizontaler Polarisation der Sendeantenne.

sind eingangs genannt. Es ist dem Verfasser ein ganz besonderes Anliegen, sich für Anregungen und Diskussionen zu bedanken, die bei den damaligen Zusammenkünften erfolgten, wobei die Zahl der Treffen wegen der politischen Lege auf ein Minimum reduziert blieb. Stellvertretend seien die Kollegen Großkopf, Fleischer, Knöpfel, Halbedel, Stepp und Gutzmann bedankt.

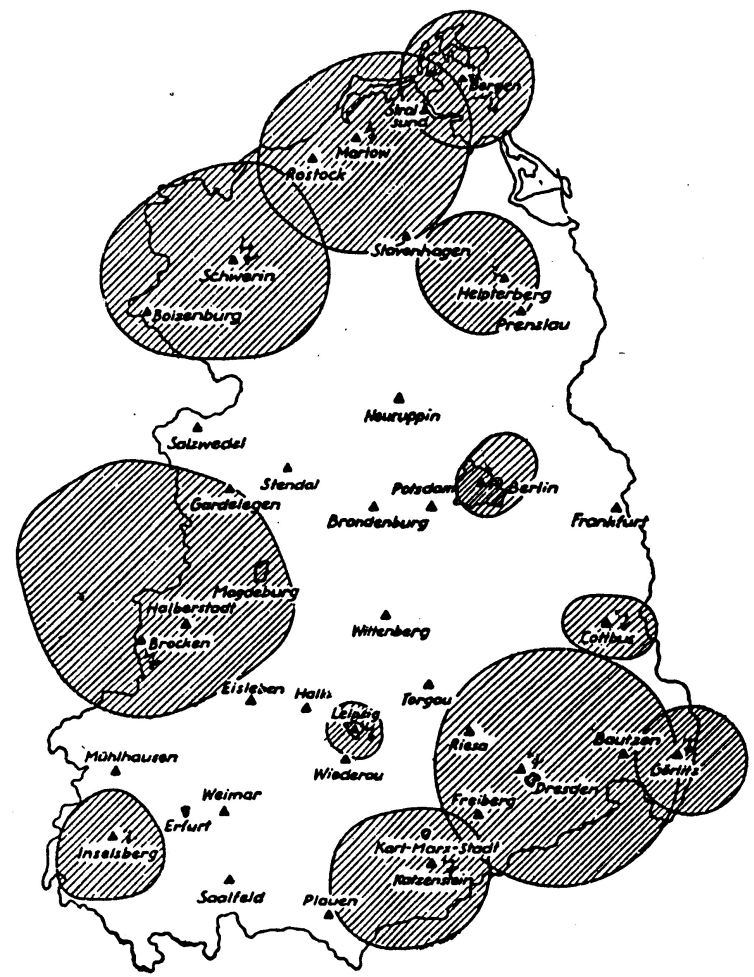

Abb. 31. Versorgung von Ostdeutschland nach OIR-Norm.

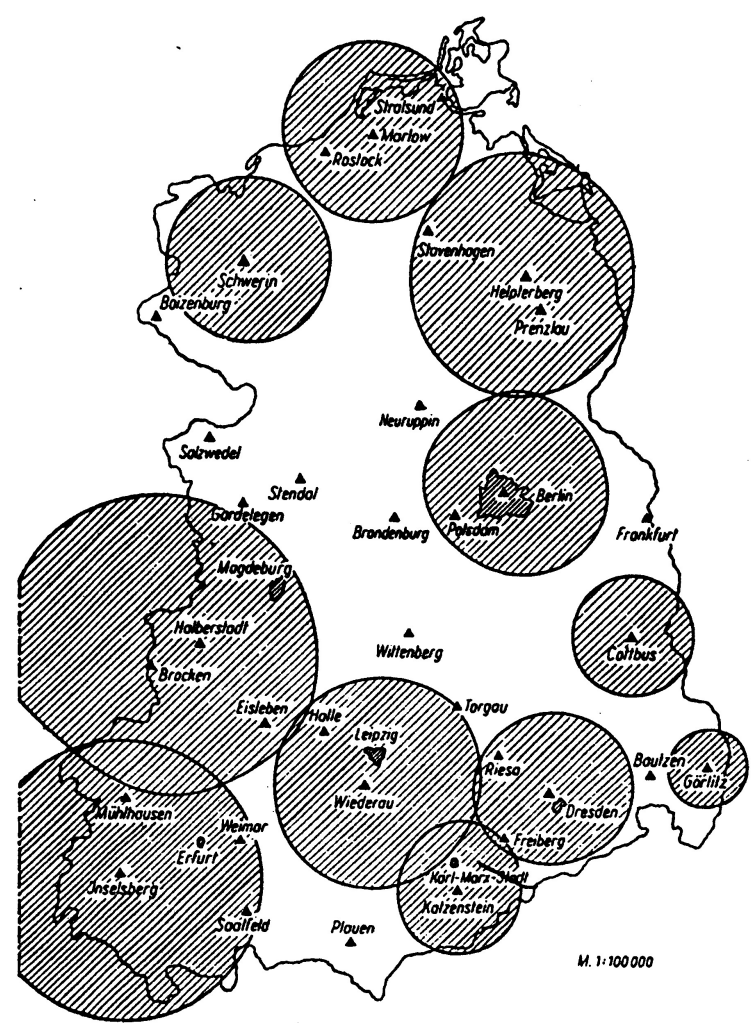

Abb. 32. Versorgung von Ostdeutschland nach CCIR-Norm. 
Allen ostdeutschen Kollegen, die damals diese Arbeiten förderten bzw. aktiv daran teilnahmen gilt gleichfalls besonderer Dank, u.a. dem wissenschaftlichen Leiter des RFZ, Prof. Stier, sowie E. Augustin ${ }^{1}$, H. Albrecht, W. Heide sowie allen meinen Mitarbeitern in Kolberg. Der Verfasser dankt vielmals Herrn Prof. Koch von der Universität Hannover für vorbereitende Diskussionen der vorliegenden Arbeit, ohne die eine Publikation nicht möglich gewesen wäre.

\section{Literatur}

Gressmann, R. and Kaltbeitzer, K. H.: Vereinfachte Verfahren zur Bestimmung der Versorgungswahrscheinlichkeit und Anwendung auf UKW-Netzplanung, Hausmittlg. des NWDR, Juni 1952.

Großkopf, J.: Die Verfahren zur Ermittlung der Versorgungswahrscheinlichkeit im Feld eines von beliebig vielen Störsendern beeinflußten Nutzsenders, Hausmittlg. des NWDR, Juni 1952.

Knöpfel, F. W. and Stepp, W.: Planung des Rundfunksendernetzes im UKW-Bereich für Hörrundfunk und Fernsehen, FTZ, H. 8, S. 535, 1953.

Králik, F., Kühn, U., and Vocaldo, V.: Ausbreitungsmessungen bei $480 \mathrm{MHz}$ und $780 \mathrm{MHz}$ im Gebirge der Niederen Tatra, Techn. Mittlg. des RFZ, 5, H. 4, S. 174, 1961.

Kühn, U.: Ausbreitungsuntersuchungen über unterschiedlichen Gelände in den Frequenzbändern I, II und III, Techn. Mittlg. des RFZ, 2, H. 1, S. 1, 1958a.

Kühn, U.: Fernsehversorgung Mitteldeutschlands nach einer neuen Frequenzplanung, Fernmeldepraxis, Bd. 55, H. 18, S. 697 , $1958 b$.

Kühn, U.: Messungen der Depolarisation bei $780 \mathrm{MHz}$ in verschiedenen Geländearten und in Städten, Techn. Mittlg. des RFZ, 6, H. 4, S. 177, 1962.

Kühn, U.: Empfangsverbesserung durch Gewitter, Radio und Fernsehen, 12, H. 1, S. 10, 1964a.

Kühn, U.: Wave propagation as a basis for the the planning of UHF transmission networks, UIT Telecommunication Journal, 31(2), p. $43,1964 b$.
Kühn, U.: Ergebnisse mehrjähriger Messungen des troposhärischen Fernfeldes bei $1.100 \mathrm{MHz}$, Kleinheubacher Berichte, FTZ, Darmstadt, Nr. 10, S. 13, 1965.

Kühn, U.: Ein Beitrag zur Frequenzabhängigkeit des Gewittereffektes, Nachrichtentechnik, 21, H. 9, S. 325, 1971.

Kühn, U. and Derer, I.: Ein Betrag zur Kenntnis der Überreichweiten an einer 377 km-Strecke bei $1.100 \mathrm{MHz}$ (Klét-Kolberg), Techn. Mittlg. des RFZ, Bd. 15, H. 2, S. 86, 1971.

Kühn, U. and Heide, W.: Der Jahresgang der UKW-Ausbreitung nach fünfjährigen Messungen in Kolberg bei Berlin, Nachrichtentechnik, 11, H. 7, S. 313, 1961.

Kühn, U. and Ogulewicz, S.: Propagation measurements at $500 \mathrm{MHz}$ over sea for varying meteorological parameters, Proc.I.E.E., 117(3), p. 879, May 1970.

Kühn, U. and Vocaldo, V.: Die Ausbreitung von VHF und UHF hinter beugenden Kanten, Mittlg. des RFZ, 16, H. 3, S. 69, 1972.

Kühn, U., Lanzke, M., and Ogulewicz, S.: Zeitliche Schwankungen beim UHF-Empfang, Techn. Mittlg. des RFZ, 17, H. 3, S. 66, 1973a.

Kühn, U., Ogulewicz, S., and Trommer, H.: UHF-Ausbreitung im Küstenbereich und über der Ostsee, Techn. Mittlg. des RFZ, 17, H. 2, S. 41, 1973b.

Nestel, W. and Schwarz, E.: Über die physikalischen Grundlagen der Senderplanung im Ultrakurzwellengebiet, Funk und Ton, H. 4, S. 165, 1953.

Ogulewicz, S., Vocaldo, V., Kühn, U., and Käbe, J.: Einige Erfahrungen mit der Fernsehversorgung im UHF-Bereich, Radio, Fernsehen, Elektronik, 22, H. 4, S. 109, 1973.

Ogulewicz, S., Kislo, M., and Kühn, U.: Ausbreitung von Dezimeterwellen an Land-Seestrecken oberhalb $300 \mathrm{~km}$ im südlichen Teil der Ostsee, Techn. Mittlg. des RFZ, 20, H. 2, S. 46, 1976.

v. Rautenfeld, F. and Fastert, H. W.: Probleme der UKWVersorgung, Techn. Hausmittlg. des NWDR, Sonderheft Juni 1952.

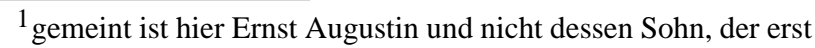
viel später im RFZ tätig war.
} 